\title{
Erkrankungen und Verletzungen der Rotatorenmanschetten
}

\author{
Sven Lichtenberg, Petra Magosch, Peter Habermeyer
}

\section{Zusammenfassung}

Mit zunehmendem Alter und steigendem Aktivitätsgrad älterer Mitbürger nimmt die Bedeutung der Erkrankungen und Verletzungen der Rotatorenmanschette stetig zu. Zum einen tolerieren die betroffenen Menschen immer seltener Einschränkungen in ihrer Leistungsfähigkeit, zum anderen steigt die Nachfrage nach konservativen und operativen Maßnahmen dieses Patientenkollektivs. Ziel dieses Artikels ist es, Ursachen, klinische Präsentation und Diagnostik zu erörtern. Ferner sollen die konservativen Maßnahmen, wie auch die Indikationen zu verschiedenen OP-Techniken diskutiert und dargestellt werden.

\section{Pathology and Lesions of the Rotator Cuff}

With increasing age and the higher functional demands of the elderly patient the importance of rotator cuff lesions and their treatment also rises. On the one hand, patients are markedly less willing to tolerate functional impairment and so that, on the other hand, the demand for optimal treatment, no matter if conservative or surgical, grows as well. The purpose of this article is to illustrate the relevant causes, clinical presentation and diagnostic measures. Furthermore, conservative treatment modalities and surgical procedures as well as their respective indications are discussed.

\section{Einleitung}

Bei zunehmendem Funktionsanspruch der in Zukunft länger im Arbeitsprozess stehenden und einer auch im Alter noch sportlich aktiven Gesellschaft kommt der Rotatorenmanschettenläsion eine hohe sozioökonomische Bedeutung zu. Gerade die Altersjahrgänge über 60 weisen in etwa einem Drittel Manschettenrupturen auf, zu deren Therapie eine differenzierte Entscheidung gefunden werden muss.

War bis zum Ende der 1990er-Jahre die offene Rotatorenmanschettenchirurgie der „golden standard“, stehen uns heute moderne arthroskopische Verfahren zur Verfügung, die mit Ausnahme der Lappenplastiken die offene Chirurgie ersetzt haben. Die jüngere Generation der orthopädischen Chirurgen hat eine hohe Affinität zur minimalinvasiven Technik

OP-JOURNAL 2013; 29: 54-70

(C) Georg Thieme Verlag KG Stuttgart · New York DOI http://dx.doi.org/10.1055/s-0032-1328431 und die Patienten wünschen ein arthroskopisches Verfahren. Dennoch ist die offene Chirurgie als Fundament zu betrachten und deren Techniken ebenso $\mathrm{zu}$ beherrschen wie die arthroskopischen. Der Chirurg, der beide Verfahren kann, verfügt über ein breiteres Therapiespektrum als der nur in einem Verfahren bewanderte.

Die Grundlagen der Rotatorenmanschettenchirurgie sind jedoch untrennbar mit dem Bostoner Chirurgen Ernest Amory Codman (1869-1940) verbunden. Die Läsionsformen der Rotatorenmanschette wurden differenziert, die klinische Relevanz der Bursa subacromialis sowie Behandlungsmöglichkeiten der Supraspinatussehnen-Partialruptur beschrieben. In seinem Werk „The shoulder“ beschreibt Codman die sog. Übergangszone, hypovaskularisierte Areale und das Spektrum der Rupturformen von artikulärseitigen Partialrupturen bis hin zu Massenrupturen.

Die sportspezifische Relevanz der Rotatorenmanschettenfunktion sowie der
Zusammenhang zwischen Rotatorenmanschettenläsionen und wiederholten Überkopfbeanspruchungen des Werfers wurden von Georg Eli Bennett (18851962) 1941 erstmals veröffentlicht.

Die Begründer der modernen Schulterchirurgie waren in Europa Didier Patte (Paris) und Norbert Gschwend (Zürich) und in New York Charles Neer. Auf Harvey Ellman (Los Angeles) geht die arthroskopische Akromioplastik Anfang der 80er-Jahre zurück. Er war es auch, der weltweit die ersten arthroskopischen Nähte an der Rotatorenmanschette durchführte.

\section{Ätiologie und Epidemiologie}

Die Prävalenz der Rotatorenmanschettenruptur in der Bevölkerung lässt sich durch autoptische Untersuchungen zwischen 5 und 39\% einordnen. Die Inzidenz bei Individuen älter als 60 Jahre mit völlig schmerzfreier, voll funktionstüchtiger Schulter liegt nach Kernspinuntersuchungen bei 22-28\%. Die Defektgröße steigt mit zunehmendem Lebensalter. Angaben über die Häufigkeit von Rotatorenmanschettenrupturen in Altersdekaden unterteilt, weisen in der Vielzahl der Veröffentlichungen eine hohe Schwankungsbreite auf. Wenn autoptische Studien auch eine Zunahme pathologischer Veränderungen mit steigendem Alter finden, besteht hier keine Korrelation zwischen diesen Befunden und der klinischen Symptomatik (Tab. 1).

Rupturen bei Jugendlichen und jüngeren Erwachsenen sind Folge eines Traumas durch Kompressions-, Scher- oder Zugkräfte höheren Ausmaßes.

Ab der mittleren Lebensdekade ereignen sich Rupturen der Supraspinatus- und Infraspinatussehne zum überwiegenden Teil auf dem Boden atraumatischer Prozesse. Programmierter Zelltod (Apoptose) der fibroblastischen Zellen lässt sich in den Sehnen der Rotatorenmanschette 
nachweisen, niedrigerer Sauerstoff- und höherer Stickstoffoxidgehalt findet sich in rupturiertem Sehnengewebe.

Häufig erleiden die Patienten bei degenerativer Schadensanlage ein akutes Trauma, das den vorliegenden Defekt vergrößert und als klinisches Bild manifest werden lässt. Die pathogenetische Beziehung zwischen Akromionsporn und Rotatorenmanschettenruptur, wie von Neer postuliert, ist nur einer von mehreren extrinsischen und intrinsischen Faktoren für Veränderungen der Rotatorenmanschette und wird heute noch deutlich von vielen überschätzt. Eine pathomechanische Bedeutung weist ein vermehrt nach lateral stehendes Akromion auf. Biomechanisch kommt es am gelenkseitigen Supraspinatussehnenansatz unter Zugbelastung zur maximalen Belastung, was den Rupturbeginn an der knöchernen Insertionszone erklärt. Eine Partialruptur erzeugt einen erhöhten Belastungsstress an der artikulärseitigen Sehneninsertionsstelle.

Die bursalseitige Partialläsion entsteht dagegen tatsächlich am häufigsten durch eine mechanische Irritation am Akromion, entweder durch eine echte knöcherne Enge durch Akromionsporn oder durch ein sekundäres Impingement bei gestörtem skapulothorakalem Rhythmus oder durch Schwäche der zentrierenden Rotatorenmanschette.

Die Grundidee einer enthesiopathischen Sehnenerkrankung am Insertionsort der Supraspinatussehne als intrinsischer Faktor des Gewebsuntergangs stammt von Uhthoff.

Hingegen werden isolierte Läsionen der Subscapularissehne zu 70\% durch ein alleiniges adäquates Trauma ausgelöst.

Die Schulterluxation führt altersabgestuft in 14-71\% der Fälle zu einer Rotatorenmanschettenruptur. Bei Patienten über 50 Jahre werden 63-71\% Rotatorenmanschettenrupturen nach Luxationsereignis berichtet.

\section{Diagnostik}

In der Anamnese ist nach einem möglichen Traum zu fragen, ferner sind Beginn der Symptome, deren Qualität und Quantität und explizit Nachtschmerzen zu erfragen.

Gerade ein Sturz auf den Ellenbogen oder die Hand mit fortgeleitetem Trauma
Tab. 1 Sonografische altersbezogene Prävalenz der Rotatorenmanschettenruptur [19].

\begin{tabular}{ll}
\hline Alter & Häufigkeit \\
\hline$<40$ Jahre & $\begin{array}{l}\text { keine partielle/komplette } \\
\text { Ruptur }\end{array}$ \\
\hline < 50 Jahre & $\begin{array}{l}\text { 5\% partielle/komplette } \\
\text { Rupturen }\end{array}$ \\
\hline$<60$ jahre & $\begin{array}{l}\text { 11\% partielle/komplette } \\
\text { Rupturen }\end{array}$ \\
\hline$>70$ Jahre & $\begin{array}{l}50 \% \text { partielle/komplette } \\
\text { Rupturen }\end{array}$ \\
\hline$>80$ Jahre & $\begin{array}{l}\text { 80\% partielle/komplette } \\
\text { Rupturen }\end{array}$
\end{tabular}

des Schultergelenks sind ebenso wie eine Luxation in der Vorgeschichte verdächtig auf eine RotatorenmanschettenLäsion.

\section{Untersuchung}

Es gibt zur Überprüfung der Funktion der RM spezielle Tests, die gesondert die einzelnen Sehnen der RM abprüfen. Neben dem nach Jobe benannten Test der SSP-Sehne (Abduktion gegen Widerstand beim um $90^{\circ}$ in der Skapulaebene abduzierten Arm, auch „empty can test“ genannt) stehen Tests zur Funktion des Subscapularis (SCP) und des Infraspinatus (ISP) zur Verfügung. Als weiterführende Literatur sei auf das Themenheft der Oberen Extremität verwiesen [1].

\section{Bildgebung}

Als primäre Bildgebung bietet sich die Ultraschalluntersuchung an, die schnell und preiswert, allerdings auch sehr untersucherabhängig ist.

Zur Röntgenstandarddiagnostik gehören:

- „true“ a.-p. Aufnahme am stehenden Patienten mit Neutralrotation des Armes

- axiale Aufnahme

- „outlet-view“

- AC-Gelenk Zielaufnahme - Zanka View - bei symptomatischem ACG-Befund

Kernspintomografien (MRT) - parakoronar, transversal und parasagittal - sollten erst dann erfolgen, wenn die Basisdiagnostik erfolgt ist und klare Fragen an den Radiologen gestellt werden können. Das MRT muss eine Aussage liefern über:
- Rupturlokalisation und -größe

- Sehnenretraktion

- muskuläre fettige Atrophie

- Begleitarthrosen (glenohumeral, ACGelenk)

- Markraumveränderung

Muskuläre Atrophien ohne Sehnenruptur müssen neurologisch mittels EMG und NLG weiteruntersucht werden.

Hinsichtlich der Schmerzentwicklung stellt sich die Frage, warum einige Patienten mit Ruptur eine klinische Symptomatik entwickeln und andere nicht. Von S. Burkhart wurde die kinematisch einwandfreie und asymptomatische Funktion des anatomisch defizienten, biomechanisch jedoch intakten Cuffs beschrieben. Asymptomatische Patienten weisen eine höhere Muskelaktivität auf als symptomatische. Eine japanische $\mathrm{Ar}$ beitsgruppe um Fukuda sieht im Grad der Bursainflammation einen ursächlichen Faktor für die klinische Relevanz der Rotatorenmanschettenruptur. Häufigste Schmerzursache ist nach unserer Meinung eine begleitende Pathologie der langen, intraartikulär verlaufenden Bizepssehne, die durch das Auftreten einer adhäsiven Kapsulitis als möglicher Schmerzfaktor verstärkt wird. Aber auch Veränderungen der glenohumeralen und skapulothorakalen Kinematik und die Unfähigkeit zur muskulären Kompensation mit Kaudalisierung des Kopfes kommen hinzu.

\section{Klassifikation}

Die Einteilung der RM-Ruptur spielt im Rahmen des präoperativen Stagings für die Planung des operativen Prozederes eine entscheidende Rolle: Um ein optimales OP-Resultat zu erzielen, muss präoperativ eine pathologiekonforme Therapieplanung durchgeführt werden. Diese beinhaltet primär auch die anamnestische Abklärung des Funktionsanspruchs des Patienten und seiner Motivation auch im Hinblick auf die langwierige postoperative Physiotherapie.

Eine arthroskopische Einteilung der Rotatorenmanschetten-Partialruptur erfolgte sowohl durch Snyder als auch von Ellman. Beide Klassifikationen weisen aber eine schlechte Intra- und Interobserver-Genauigkeit bezüglich der artikularseitigen Defektausdehnung auf.

Eine neue Einteilung der Partialrupturen erfolgt nach Habermeyer [2] (Abb.1). Hierbei wird die Supraspinatussehne 


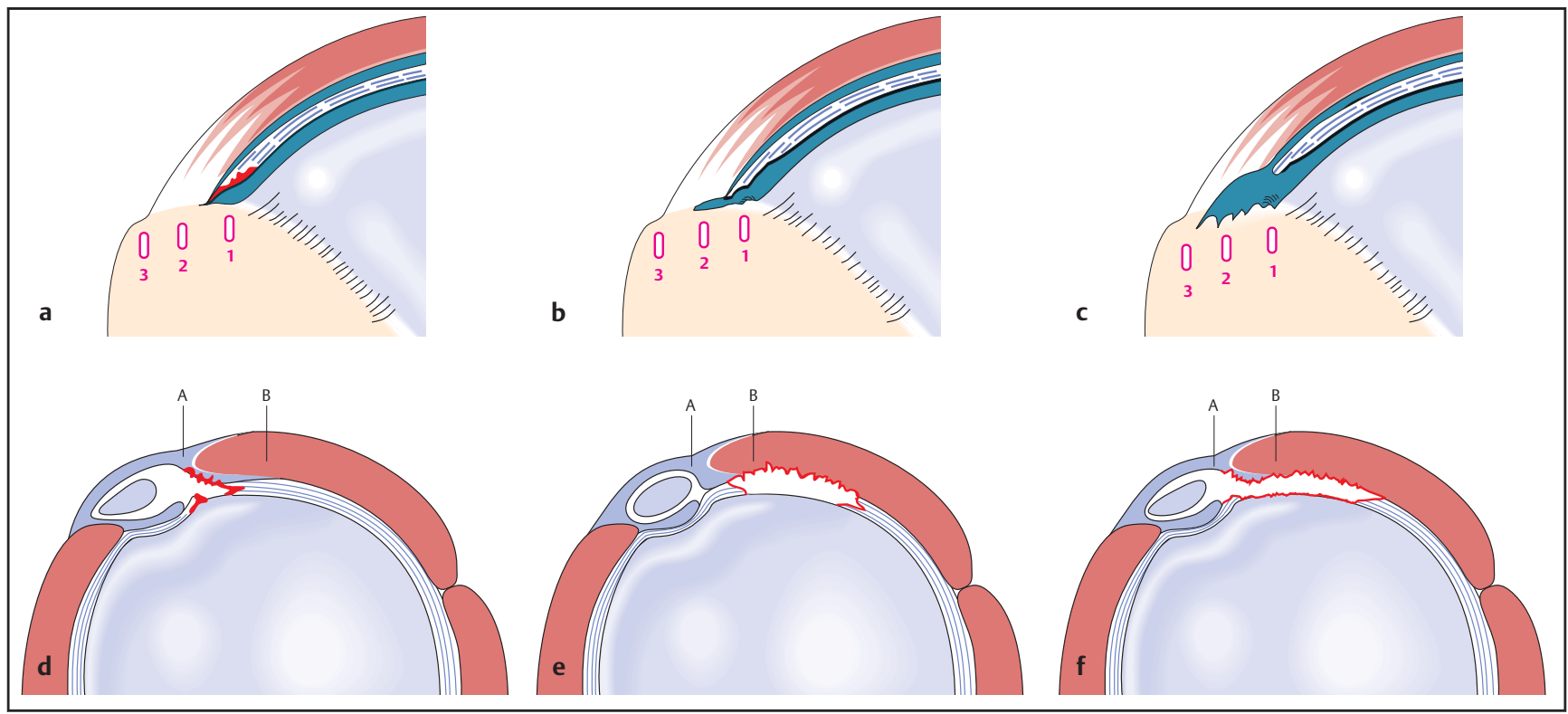

Abb. 1 Klassifikation der SSP-Partialruptur nach Habermeyer. a-c Longitudinale Ausdehnung der artikularseitigen Supraspinatussehnenpartialruptur in der parakoronaren Ebene. a Typ-1-Ruptur: kleine Ruptur in der Übergangszone von Knorpel zu Knochen. b Typ-2-Ruptur: Ausdehnung der Ruptur bis zur Mitte des Footprints. c Typ-3-Ruptur: Ausdehnung der Ruptur bis zum Tuberculum majus. d-f Sagittale Ausdehnung der artikularseitigen Supraspinatussehnenpartialruptur in der transversalen Ebene (A: Typ A, B: Typ B). d Typ-1-Ruptur: Ruptur des lateralen Anteils des Lig. coracohumerale mit Ausdehnung in den medialen Supraspinatussehnenansatz. e Typ-2-Ruptur: isolierte Partialruptur der Suparspinatussehne innerhalb der Crescent-Zone. f Typ-3-Ruptur: Ruptur, die sich vom lateralen Anteil des Lig. corachumerale über den medialen Supraspinatussehnenansatz hinweg bis in die Crescent-Zone fortsetzt (Mit freundlicher Genehmigung aus: Habermeyer, Lichtenberg, Magosch. Schulterchirurgie. (c) Elsevier $\mathrm{GmbH}$ ).

(SSP) in der parasagittalen Ebene in die Sektoren A, B und C unterteilt:

- Zone A beschreibt die laterale PulleySchlinge, die direkt medial distal in die SSP einmündet.

- Zone B beschreibt die sog. „crescent zone“, die den sehnigen Übergang zur Insertionszone darstellt.

- Zone C ist die Kombination aus Zone A und $\mathrm{B}$.

In der parakoronaren Ebene werden die artikularseitigen Rupturen eingeteilt in die Typen I, II und III:

- Typ I bezeichnet einen Partialdefekt, der sich nur auf den chondralen Übergang zur Insertionszone erstreckt.

- Typ II beschreibt die weitere Ausdehnung nach lateral bis zum Zentrum des sog. „footprint“,

- Typ III die komplette Ausdehung bis zum Tuberculum majus.

Die durchschnittliche Sehnenbreite in parasagittaler Richtung beträgt $25 \mathrm{~mm}$ und $12 \mathrm{~mm}$ in der koronaren Schicht. Liegt der Sehnenansatz artikularseitig etwa $6 \mathrm{~mm}$ frei vom knöchernen Sehnenlager, kann man von einer 50\%igen Teilruptur ausgehen.

Eine Sonderform stellt hier die intratendinöse Ruptur zwischen den Sehnenblättern dar: Sehnenunter- und -oberflä- che sind hierbei intakt. Die Diagnose gelingt meist nur mithilfe des MRT. Hinsichtlich der Inzidenz fand Fukuda in 149 Schulterpräparaten 13\% partielle intratendinöse Rotatorenmanschettenrupturen.

Die Rupturgröße bei kompletten Läsionen wird hinsichtlich ihrer Größe nach Bateman eingeteilt, wobei die größte Defektbreite am Insertionsort humeralseitig bestimmt wird.

Grad I: $<1 \mathrm{~cm}$

Grad II: $1-3 \mathrm{~cm}$

Grad III: $3-5 \mathrm{~cm}$

Grad IV: $>5 \mathrm{~cm}$

Die Klassifikation der Rupturform nimmt Bezug auf Form und Größe und besitzt daher eine wichtige prognostische Aussagekraft.

- halbmondförmige (crescent-shape) Ruptur: Ruptur des SSP mit leichter Retraktion, ähnlich dem Rotatorenkabel

- U-förmige Ruptur: große Ruptur von SSP und ISP mit einsprechend stärkerer Retraktion vor allem nach anteromedial und posteromedial, sodass ein großes U-förmiges Loch entsteht

- L-förmige oder umgedreht L-förmige Ruptur: Ruptur des SSP vom Footprint mit zusätzlicher longitudinaler Kom- ponente zwischen SSP und RI oder SSP und ISP. Retraktion des Haupsehnenanteils stets weg von der longitudinalen Komponente

- Massenruptur: Ruptur von 3 Sehnen

Hinsichtlich einer prognostischen Wertung bedeutsam ist die Einteilung der Rotatorenmanschettenruptur nach ihrer Anamnesedauer.

- akut: <6 Wochen

- subakut: 6 Wochen - 6 Monaten

- chronisch: 6 Monate - 1 Jahr

- alt: > 1 Jahr

Als weiterer prognostischer Faktor lässt sich präoperativ im MRT die Sehnenretraktion sowie die Muskelatrophie untergliedern (Tab. 2).

\section{Prognose}

Defektgröße und Anamnesedauer beeinflussen das operative funktionelle Ergebnis.

Hinsichtlich der Größe der Rotatorenmanschettenruptur und der operativen Prognose besitzen bereits folgende klinische Zeichen eine negativ prädiktive Bedeutung:

- Kraftverlust bei Abduktion, insbesondere bei Außenrotation des horizontal 
Tab. 2 Beurteilung der Muskelatrophie und Sehnenretraktion [20,21].

\begin{tabular}{|c|c|c|}
\hline Sehnenretraktion n. Patte & Atrophie im CT & Atrophie im MRT \\
\hline $\begin{array}{l}\text { I: Stumpf zwischen Tuber- } \\
\text { culum majus und Apex }\end{array}$ & I: normal & $\begin{array}{l}\text { I: normal oder leicht atro- } \\
\text { phierter Muskel, Okkupations- } \\
\text { verhältnis der Fossa supraspi- } \\
\text { nata } 0,66-1,0\end{array}$ \\
\hline $\begin{array}{l}\text { II: Stumpf zwischen Apex } \\
\text { und Glenoid }\end{array}$ & $\begin{array}{l}\text { II: intramuskuläres Fett } \\
<\text { Muskelvolumen }\end{array}$ & $\begin{array}{l}\text { II: mäßige Atrophie, Okkupa- } \\
\text { tionsverhältnis der Fossa } \\
\text { supraspinata } 0,4-0,6\end{array}$ \\
\hline $\begin{array}{l}\text { III: Stumpf medial des } \\
\text { Glenoids }\end{array}$ & $\begin{array}{l}\text { III: intramuskuläres Fett } \\
=\text { Muskelvolumen }\end{array}$ & $\begin{array}{l}\text { III: deutliche oder schwere } \\
\text { Atrophie, Okkupationsverhält- } \\
\text { nis der Fossa supraspinata } \\
<0,4\end{array}$ \\
\hline & $\begin{array}{l}\text { IV: intramuskuläres Fett } \\
>\text { Muskelvolumen }\end{array}$ & \\
\hline
\end{tabular}

gestreckten Armes (Patte-Test entspricht „full can“-Test)

- Atrophie der Supra- und Infraspinatusmuskulatur

- vordere Schultersubluxation bei Abduktion (Subscapularisbeteiligung)

- vorbestehende spontane Ruptur der langen Bizepssehne als Zeichen fortgeschrittener degenerativer Veränderungen

- Einschränkung der passiven Gelenkbeweglichkeit

Prognostische radiologische Zeichen für das OP-Ergebnis ist die Korrelation mit dem präoperativ gemessenen akromiohumeralen Abstand (AHA) auf der Röntgennativaufnahme (,true a.-p.“-Aufnahme beim stehenden Patienten, mit angelegtem Oberarm bei $0^{\circ}$-Neutralstellung). Beträgt der Abstand $8 \mathrm{~mm}$ und mehr, kann ein günstiges operatives Ergebnis erwartet werden. Bei einem Abstand zwischen 5 und $7 \mathrm{~mm}$ muss man einen schwierigen Verschluss der Ruptur erwarten (Abb. 2). Darunter ist kein kompletter Defektverschluss mehr möglich. Ein Os acromiale gilt nach unserer Meinung als Ursache für erhöhte Re-Rupturgefahr.

Der wichtigste Outcome-Faktor ist letztlich die Rupturgröße, mit welcher das Alter des Patienten korreliert [3,4]. Mit Zunahme der Rupturgröße kommt es zum Rückgang der reparativen Vorgänge und zur vermehrten chondroiden Metaplasie und Amyloideinlagerung [5]. Bei veralteten, großen Sehnenrupturen mit weiter Retraktion (Patte $3^{\circ}$, d.h. bis an den Glenoidrand) und manifester fettiger Infiltration und Atrophie Grad $\mathrm{III}^{\circ}$ und $\mathrm{IV}^{\circ}$ ist mit einer hohen Versagensquote zu rechnen.
Hinsichtlich der Lokalisation hat die isolierte Supraspinatussehnenruptur die günstigste Prognose, die Kombination einer Ruptur der Supraspinatussehne mit der Infraspinatussehne ist günstiger als die mit der Subscapularissehne. Am ungünstigsten und auch operativ am schwierigsten $\mathrm{zu}$ verschließen ist die Ruptur aller 3 Sehnen, wobei die zusätzliche Läsion der langen Bizepssehne eine weitere Verschlechterung der Prognose bedeutet.

Auftreten und Progression der fettigen Muskelatrophie korreliert mit der Zahl der gerissenen Sehnen und dem steigenden Patientenalter. Rotatorenmanschetten entwickeln in unterschiedlicher Geschwindigkeit ihre fettige Atrophie in Abhängigkeit vom Beginn der Symptome, wobei der Subscapularis am schnellsten atrophiert.

Die fettige Infiltration und Atrophie speziell der Infraspinatusmuskulatur beinflussen das funktionelle Outcome. Durch Verkleinerung der Muskelquerschnittsfläche reduziert sich die Zugkraft des Muskels (max. 200 N). Selbst eine erfolgreiche Naht führt zu keiner Verbesserung des Verfettungsgrads und nur zu einem leichten Rückgang der Atrophie, hingegen eine Re-Ruptur zu deren Progression.

Der Grad der Retraktion des Sehnenstumpfs und der damit verbundenen Verkürzung des Muskels führt zu irreversiblen Veränderung der Muskelstruktur mit Atrophie, Infiltration von Fettzellen und Zunahme von interstitiellem Bindegewebe mit Störung der physiologischen Eigenschaften des Muskelgewebes. Der Zeitpunkt zur Operation muss vor Eintreten der muskulären Verän-

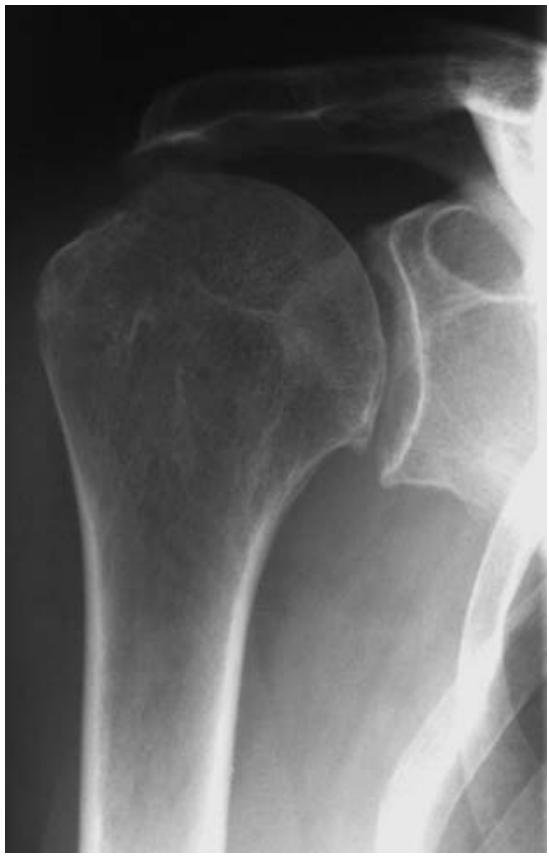

Abb. 2 True a.-p. Röntgenbild mit vermindertem akromiohumeralen Abstand.

derungen, d.h. vor 6 Wochen liegen. Die intraoperative Überprüfung der Verschiebbarkeit, d.h. Elastizität des Sehnen- und Muskelgewebes, ist eine wichtige prognostische Beurteilungsgröße. Lässt sich die fibröse Verkürzung der Muskulatur nicht mehr mobilisieren, macht es aus muskelphysiologischen Gründen keinen Sinn mehr, durch Verschiebeplastiken einen Verschluss zu erzwingen, weil die kontraktilen Muskelelemente irreversibel geschädigt sind und nur ein Tenodese-Effekt erreicht wird.

Die Qualität des Sehnengewebes beeinflusst als mechanischer Faktor die Qualität des Einheilungsergebnisses.

Raucher weisen ein höheres Risiko für Rotatorenmanschettenrupturen auf, zudem sind die Operationsergebnisse schlechter. Nikotin verzögert experimentell die Sehneneinheilung. Diabetes mellitus ist mit einer höheren Inzidenz an postoperativen Infekten vergesellschaftet, zudem besteht eine höhere Gefahr für das Auftreten einer postoperativen Frozen Shoulder. Rentenbegehren und BG-liche Unfälle haben erfahrungsgemäß zumindest subjektiv schlechte Resultate.

\section{Konservative Behandlung der Rotatorenmanschettenruptur}

Prinzipiell ist abzuwägen zwischen möglichem konservativen Behandlungserfolg und der Gefahr einer Umwandlung eines einfachen Sehnenrisses in eine irrepara- 
ble Situation durch die konservative Behandlung. Hierbei spielen zum einen „weiche“ Kriterien wie Patientenalter und -anspruch sowie grundsätzlicher Gesundheitszustand eine Rolle. Zum anderen müssen aber auch Aktualität einer Schädigung und einer Funktionseinbuße mit dem notwendigen Funktionsanspruch eines körperlich berufstätigen Menschen in den Entscheidungsprozess miteinbezogen werden.

Der verständliche Wunsch des Patienten, sich einem operativen Eingriff zu entziehen, gepaart mit unkritischer abwartend konservativer Haltung des behandelnden Arztes führt zu einer Verschlechterung der Ausgangssituation mit einem verspätet nicht mehr einfach rekonstruierbaren Defekt und dann weiterreichendem operativen Verfahren. Mindestens 50\% der zunächst asymptomatisch behandelten konservativen Fälle entwickeln nach einer epidemiologischen Studie und einer Längsschnittbeobachtung zunehmende Beschwerden und eine Größenzunahme des Defekts.

Das klinische Beschwerdebild ist different. Kleinere Einrisse der Manschette können asymptomatisch verlaufen und zu keiner relevanten Einschränkung im Alltag führen, wobei sie jedoch mit zunehmender Rupturgröße symptomatisch werden können. Dementgegen konnten Matthews et al. [5] zeigen, dass Partialrupturen und kleine Totalrupturen mit einem nachweisbaren Regenerationsprozess einhergehen. Neben einer vermehrten Gefäßeinsprossung fanden die Autoren eine erhöhte Zahl an Entzündungszellen und Fibroblasten.

Arbeiten beschreiben eine Verschlechterung im SF36 General Health Test im Verlauf der Beobachtungszeit. Patienten aus niedrigen sozialen Schichten zeigen ein signifikant schlechteres Resultat nach konservativer Therapie. Welche klinischen Zeichen mit dem Endergebnis korrelieren untersuchte Itoi in einer Untersuchung bei 62 Patienten mit kompletter Ruptur. Dabei zeigten sich als Indikatoren für das Langzeitergebnis der Grad und die Kraft bei der Abduktion. Je besser und kraftvoller die Patienten bei der Erstuntersuchung abduzieren konnten, desto besser das Endergebnis. Hinsichtlich der Lokalisation hat die isolierte Supraspinatussehnenruptur die günstigste Prognose. Der Anteil des Supraspinatus an der Abduktions- und Außenrotationskraft beträgt in einer In-vivo-Untersuchung zwischen 20 und 30\%. Insbe- sondere Langzeitstudien machen deutlich, dass es sich bei konservativer Therapie nicht um dauerhafte positive Resultate handelt. Zwar können konservativ behandelte Rotatorenmanschettenmassenrupturen bis zu 4 Jahre klinisch kompensiert bleiben, radiologisch zeigt sich aber eine progressive degenerative Gelenkschädigung. Nach 4 Jahren wird aus einem rekonstruierbaren ein nicht wieder herzustellender Defekt. Nach Auswertung der Literatur zeigt sich insgesamt, dass es nur in 50\% zu einer zufriedenstellenden Schmerzbefreiung und zu keiner Kraftzunahme im Langzeitverlauf kommt. Im Vergleich dazu erreichen operierte Patienten 85\% Schmerzfreiheit und eine Verbesserung der Kraft.

Die Entscheidung hinsichtlich eines konservativen Vorgehens allein vom Patientenalter abhängig zu machen, wurde in den letzten Jahren mehr und mehr verlassen, da postoperative Ergebnisse vom Patientenalter weniger abhängig zu sein scheinen als bisher angenommen. Im Alter nimmt die Rupturgröße zu, welche wiederum das Operationsergebnis beeinflusst. Mit zunehmender Lebensdauer und vor allem -aktivität sowie deutlich steigendem Funktionsanspruch kann auch bei älteren Patienten über 70 Jahre noch die Indikation zur Operation bestehen. Hier erfordert die Rehabilitation zwar mehr Zeit und die Rezidivrate ist etwas höher, aber das funktionelle Ergebnis und die Zufriedenheit der Patienten mit dem Ergebnis sind durchaus vergleichbar mit der Rekonstruktion bei Jüngeren.

Eine begleitende adhäsive Kapsulitis stellt insofern eine Besonderheit dar, da sie unabhängig der Rupturgröße und deren Dekompensation primär ein konservatives Vorgehen bis zum Erreichen eines passiv nahezu freien Bewegungsausmaßes erfordert, da sie die Prognose des operativen Therapieerfolgs sonst deutlich verschlechtert.

Im Rahmen der diagnostischen Abklärung ist ein rationelles Vorgehen $\mathrm{zu}$ empfehlen:

Als Erstdiagnostik, die auch vom praktischen Arzt durchgeführt werden kann, erfolgen neben der klinischen Untersuchung die Röntgendiagnostik (a.-p. und axial) und, falls vorhanden, die Ultraschalluntersuchung. Besteht die Verdachtsdiagnose einer Ruptur, erfolgt die sofortige Überweisung zum Facharzt.
Spätestens 6 Wochen nach konservativer Behandlung mit - wenn auch rückläufiger - Beschwerdesymptomatik muss durch Ultraschall und im Zweifel durch Kernspintomografie die Diagnose der Manschettenruptur erbracht werden.

Die konservative Behandlung von Rotatorenmanschettenrupturen muss ebenso wie die operative strengen Indikationskriterien unterliegen:

Als Kriterien für eine konservative Behandlung können herangezogen werden:

- geringe bis fehlende Schmerzen

- langsamer Beginn

- degenerative Genese

- nicht dominante Seite

- inaktiver, alter Patient

- Non-Compliance

- begleitende adhäsive Kapsulitis

- Begleiterkrankungen wie Osteoporose, Diabetes mellitus, rheumatoide Arthritis

- längere Cortisoneinnahme

Ein Abbruch der konservativen Therapie ist zu fordern bei:

- Schmerzpersistenz über 6 Wochen

- Drop Arm Sign mit aktivem Abduktionsverlust über 6 Wochen

- funktionelle Verschlechterung nach anfänglicher Funktionserholung

\section{Kontraindikationen für konservative Therapie sind}

- akutes Trauma der Rotatorenmanschette mit fehlender Schadensanlage

- primäre Subscapularis- und Infraspinatusbeteiligung (2 Sehnenrupturen) wegen hoher Retraktionsgefahr

- Ruptur bei traumatischer Schulterluxation

- aktiver Patient im Arbeitsleben bei gesicherter Compliance

- hoher Funktionsanspruch in Sport und Freizeit

- Hemiplegie der unteren Extremität (Rollstuhlfahrer) oder Paraplegie des kontralateralen Arms

\section{Operative Therapie}

Die Indikation zum operativen Vorgehen ergibt sich aus den Kontraindikationen zum konservativen Vorgehen. Alle frischen, reparablen Läsionen der Rotatorenmanschette mit Funktionseinschränkung des aktiven Patienten sollten heute einer operativen Rekonstruktion zugeführt werden, um ein möglichst optimales Ergebnis für den Patienten erwarten 
zu lassen. Ferner müssen diejenigen Rupturen, die den Patienten auch trotz konservativer Therapie nach 3 Monaten weiterhin chronische, vor allem aber nächtliche Schmerzen bescheren, einer operativen Therapie zugeführt werden.

\section{Absolute Indikationen}

- alle traumatische Rupturen insbesonders Subscapularissehnenruptur

- Rotatorenmanschettenrupturen nach Schulterluxation

- degenerative 2-Sehnen-Rupturen (SSP + ISP oder SSP + SCP)

- degenerative Supraspinatussehnenruptur mit Schädigung der langen Bizepssehne bei Pulley-Läsion

- degenerative Suprasinatussehnenruptur mit begleitendem Os acromiale

Die alleinige subakromiale Dekompression bei kompletten Rupturen kann in ihren Langzeitresultaten nicht überzeugen.

\section{Relative Indikationen}

- Supraspinatussehnenpartialruptur $>50 \%$ (artikulär - bursalseitig)

- degenerative Supraspinatussehnenruptur ohne LBS-Schädigung

- Sonderfall: schmerzfreie, klinisch funktionell kompensierte Supraspinatussehnenruptur

- ältere funktionell anspruchslose Patienten mit Komorbidität werden konservativ behandelt.

- Jüngeren sportlichen Patienten empfehlen wir wegen der obligaten fettigen Muskelatrophie und der Progression der Defektgröße die Rekonstruktion.

\section{Patienten älter als 65 Jahre}

- frische traumatische Sehnenrupturen bei bis dato intakter Rotatorenmanschette

- frische traumatische Rupturen der Subscapularis- oder Infraspinatussehne bei bisher klinisch inapparenter, kompensierter Supraspinatussehnenläsion („Zusatz-Ruptur“, bzw. akutauf-chronisch)

- frische traumatische Sehnenrupturen ohne Humeruskopfhochstand und Muskelatrophie

Im MRT muss zwischen posttraumatischem Ödem mit Flüssigkeitseinlagerung in die Muskulatur und echter fettiger Muskelatrophie beim chronischen Defekt unterschieden werden.
Die Sehnenretraktion stellt sich besonders bei Beteiligung von 2 Sehnen innerhalb kürzester Zeit ein und kann nicht als Unterscheidungsmerkmal herangezogen werden. Ferner ist die Diskrepanz zwischen kernspintomografisch vermuteter Retraktion (bedingt durch schräge Retraktion der Sehnenstrukturen) und wahrer Retraktion unterschieden werden.

Nur durch die Beurteilung der MRT-Aufnahmen in 3 Ebenen und aufgrund der klinischen Erfahrung des Orthopäden kann hier sicher differenziert und eine voreilig als irreparabel eingeschätzte Ruptur sicher beurteilt werden.

\section{Kontraindikationen}

Die operativen Rekonstruktionstechniken unterliegen in ihren Indikationen einer Reihe von Einschränkungen.

Allgemein gilt, dass ab einer drittgradigen Sehnenretraktion nach Patte und bei einer fettigen Atrophie im Stadium Goutallier Grad III und IV ${ }^{\circ}$ eine chronisch-degenerative 2-Sehnen-Ruptur nicht mehr erfolgreich rekonstruiert werden kann!

\section{Absolute Kontraindikationen}

- florider Infekt

- Parese des N. suprascapularis oder des Armplexus

- Algodystrophie der Schulter

- Defektarthropathie der Schulter mit AHA $<5 \mathrm{~mm}$

\section{Relative Kontraindikationen}

- schmerzhafte begleitende adhäsive Kapsulitis: erfordert eine kurzfristige systemische Cortisontherapie mit begleitender manueller Therapie vor definitiver Operation

- Begleiterkrankungen mit Disposition zur Re-Ruptur: chronische Cortisoneinnahme, schwere Osteoporose

- mangelnde Compliance

- Alkoholkrankheit, Nikotinabusus

\section{OP-Zeitpunkt}

Neben den schon erwähnten Prognosefaktoren spielt auch der Zeitpunkt der OP eine wesentliche Rolle. So zeigen sich bei der traumatischen Ruptur die idealen Resultate nach einer Versorgung innerhalb der ersten 6 Wochen, zumindest sollte die Anamnesedauer 4-6 Monate nicht überschreiten. Besonders
Subscapularisrupturen sollten frühestmöglich operiert werden, da sich mit zunehmender Anamnesedauer die Resultate deutlich verschlechtern, was an der erheblichen Retraktion der Subscapularissehne liegt.

Diese Richtlinien sollten auch für traumatische Abrisse des älteren Menschen (>65) gelten. Deren funktionelles Ergebnis und die Zufriedenheit der Patienten mit dem Ergebnis sind durchaus vergleichbar mit der Rekonstruktion bei Jüngeren.

Eine Conditio sine qua non für eine komplikationslose postoperative Ausheilung ist die präoperativ freie passive Beweglichkeit. Diese muss durch hochdosierte, aber kurzfristige Gabe steroidaler Antiphlogistika und manuelle Therapie präoperativ hergestellt oder zumindest deutlich verbessert werden. Ausnahme hierzu stellt die frische, akute traumatische Sehnenruptur dar. Diese kann auch bei akut, nach dem Trauma aufgetretener Bewegungseinschränkung problemlos primär akut operiert werden.

\section{Präoperative Patientenaufklärung}

Die Rotatorenmanschettenrekonstruktion ist mit einer geringen Inzidenz an Komplikationen behaftet und im Rahmen der schulterchirurgischen Eingriffe ein sicheres Verfahren.

Beim Aufklärungsgespräch ist dem Patienten der Defektzustand sowie das operative Vorgehen anhand schematischer Zeichnungen oder Modelle zu erklären und zu dokumentieren. Die Alternative einer konservativen Behandlung mit der im Vergleich schlechteren Schmerzbefreiung und einer fehlenden Kraftverbesserung müssen aufgezeigt werden

Hinsichtlich möglicher Komplikationen unterscheiden wir zum einen die intraund postoperativen Komplikationen sowie zum anderen seltenere, oft schicksalhafte Verläufe von häufigeren und oftmals vom Patienten beeinflussbaren Komplikationen. Auf das Management der Komplikationen soll gesondert eingegangen werden.

Die wichtigsten intraoperativen Komplikationen sind:

- Akromionfraktur bei fehlerhafter Akromioplastik 
- zu ausgedehnte Resektion des Akromions mit resultierender Deltaschwäche

- Übersehen eines instabilen Os acromiale

- zu ausgedehnte Resektion der lateralen Clavicula mit Instabilität des ACGelenks

- Verletzungen des N. suprascapularis und N. axillaris

Zu den postoperativen Komplikationen zählen:

- persistierendes subakromiales Impingement

- Re-Ruptur

- Sehnenrandnekrose

- Infektion

- Nachblutung

- adhäsive Kapsulitis und restriktive subdeltoidale Adhäsionen [6]

- Kraftdefizit

- heterotope Ossifikationen [7]

- Nahtinsuffizienz des M. deltoideus bei offenen Verfahren

Die Möglichkeit einer Re-Ruptur stellt aber keine formale Kontraindikation für eine Sehnenrekonstruktion dar, wenn eine funktionelle Wiederherstellung erzielt werden soll. Selbst bei Eintritt einer Re-Ruptur liegen die Ergebnisse hochsignifikant über dem präoperativen Ausgangsbefund, wie eigene Studien bewiesen haben [8].

Insbesondere im Hinblick auf die vergleichsweise häufige Anzahl der adhäsiven Kapsulitiden ist besonderer Wert auf eine ausreichende Aufklärung der langwierigen und intensiven Nachbehandlung zu legen.

Der Patient muss sich darüber im Klaren sein, dass er und sein Physiotherapeut einen nicht unerheblichen Anteil zum Endresultat beitragen.

Nur so kann er die zum Teil 6 Monate dauernde Zeit des Heilungsverlaufs erfolgreich bewältigen.

\section{Infektionsprophylaxe und \\ Thromboseprophylaxe}

Die gezielte präoperative Antibiotikaprophylaxe vor arthroskopischen und offenen Rekonstruktionen wird allgemein anerkannt. Die häufig gefundenen Keime sind Staphylococcus aureus, Coagulasenegativer Staphyloccocus und Propionibacter acnes species. Aufgrund der typischen Empfindlichkeit ist Cefazolin als das Cephalosporin der 1. Generation am

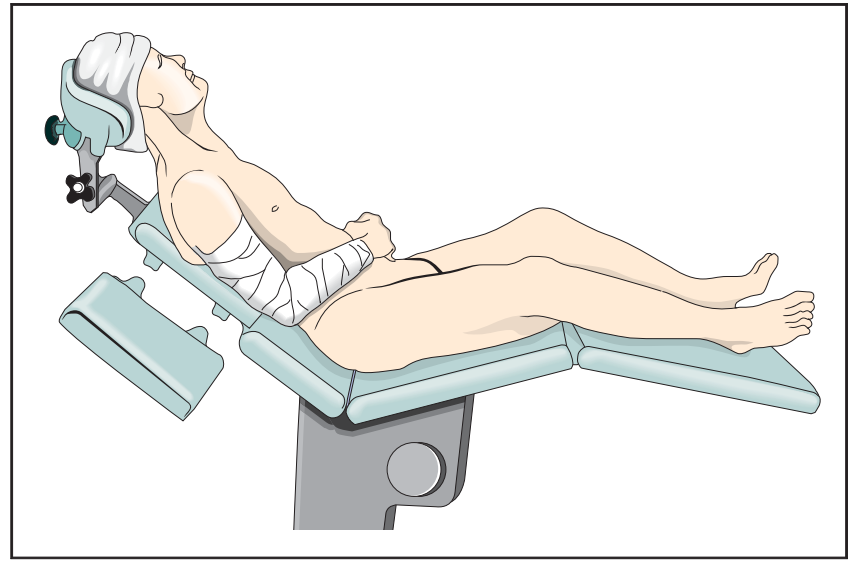

Abb. 3 Lagerung des Patienten in Beach-Chair-Position auf einem Spezialtisch mit heraus nehmbarem Schulterteil, sodass ein freier Zugang zum Schultergelenk möglich ist (Mit freundlicher Genehmigung aus: Habermeyer, Lichtenberg, Magosch. Schulterchirurgie. (c) Elsevier $\mathrm{GmbH})$.

häufigsten in Anwendung. Weitere Arbeiten legen nahe, dass ein Kombinationspräparat aus Amoxicillin und Sulbcatam $\left(\right.$ Unacid $^{\circledR}$ ) insbesondere gegen die kritischen Propioni-acnae-Bakterien wirksam ist.

Obgleich im angloamerikanischen und französischen Sprachraum nicht üblich, empfehlen wir die subkutane Injektion von niedermolekularem Heparin mit der Narkoseeinleitung. Auf Thromboseprophylaxestrümpfe verzichten wir bei der Rotatorenmanschettenrekonstruktion gänzlich.

\section{Lagerung und Narkose}

Wir empfehlen zur Versorgung der Rotatorenmanschette eine Intubationsnarkose mit gleichzeitigen interskalenären Block zur postoperativen Schmerzbefreiung. Die im Hause bevorzugte Lagerung ist die Beach-Chair-Lagerung (Abb. 3).

Der Anästhesist sollte versuchen, einen systolischen Maximalblutdruck von $100 \mathrm{mmHg} z u$ halten, soweit dies mit der zerebralen Funktion des Patienten vereinbar ist. Die Seitenlage ist für diesen Eingriff ebenfalls möglich, im eigenen Vorgehen jedoch nicht präferiert.

Jeder Eingriff, auch bei geplantem Muskel-Sehnen-Transfer, beginnt zweifelsfrei und in jedem Fall mit der diagnostischen Arthroskopie. Nur so lassen sich intraartikulär Aussagen zur Partialruptur, Bizepssehnenpathologie, Rotatorenintervallläsionen, Labrumläsionen, Knorpelschädigung und Kapsulitis stellen. Wird sie nicht durchgeführt, so ist das ein Kunstfehler.

Mit der diagnostischen Gelenkspiegelung wird zudem die OP-Indikation überprüft und gegebenenfalls die ge- plante OP-Technik umgestellt. Arthroskopisch überprüft man:

- die Sehnenqualität

- die Sehnenretraktion

- die Rupturform und Rupturgröße

- die Mobilisierbarkeit

Es darf nicht sein, dass die Schulter eröffnet wird und man dann erst feststellt, dass der Defekt nicht mehr verschließbar ist.

Subakromial beurteilt man das Ausmaß der Bursitis, des Akromionsporns und der AC-Gelenk-Arthrose und die Mobilität eines etwaigen Os acromiale.

Heutzutage hat sich, ähnlich der Kreuzbandchirurgie, die arthroskopische Rekonstruktion der Rotatorenmanschette als Standardverfahren etabliert.

Es ist aber nicht als Kunstfehler zu betrachten, wenn eine arthroskopische RM-Rekonstruktion aufgrund technischer Probleme in eine Mini-openoder offene Vorgehensweise verändert wird. Die Ergebnisse des rein arthroskopischen und der verschiedenen offenen Verfahren zeigen keine signifikanten Unterschiede im Outcome. Dies ist bis jetzt auch nicht zwischen den Single-Rowund Double-Row-Rekonstruktionen $\mathrm{zu}$ beobachten.

Zu einer zufriedenstellenden Versorgung von Läsionen der Rotatorenmanchette gehört obligat - je nach Schädigungsschwere - die Versorgung der langen Bizepssehne (LBS), des Subakromialraums mit subakromialer Dekompression und des Schultereckgelenks (laterale Clavicularesektion). Die Indikationen zu diesen einzelnen additiven Eingriffen ergeben sich aus der klinischen Symptomatik und dem etwaigen Lokalanästhesie-Test. 


\section{Partialläsionen}

Heute ist die Versorgung der artikulärseitigen Partialläsionen eine Domäne der Arthroskopie. Die arthroskopische Rekonstruktion der Sehne zeitigt gute Ergebnisse, wie dies von den Arbeitsgruppen um Yamaguchi und Castagna gezeigt wurde $[9,10]$. Bei hochgradigen Partialläsionen führte die Konversion der Läsion in eine komplette Ruptur mit anschließender Rekonstruktion zu einer kompletten Einheilungsrate von $88 \%$. Die Autoren gaben vor allem das Alter als kritischen prognostischen Faktor an, da das Durchschnittsalter der Patienten mit persistierendem Defekt mit 63 Jahren signifikant höher war als das derer mit intakter Sehne (52 Jahre). Castagna et al. [9] berichten über gute Ergebnisse in $98 \%$ von 54 Patienten mit einer transtendinösen Technik (s.u.). Eine größere Retraktion des artikulärseitigen Rupturblatts war ein prognostischer Faktor für ein schlechteres Ergebnis, wohingegen bessere Resultate bei einem größeren freiliegendem Footprint-Areal, bei geringerem Alter und bei traumatischer Genese zu beobachten waren.

Technik der arthroskopischen

Rekonstruktion der artikulärseiteigen

Supraspinatuspartialläsion

Bei der arthroskopischen Versorgung der artikulärseitigen Partialläsion des Supraspinatus kann man entweder den Defekt in situ rekonstruieren oder die Läsion komplettieren und dann wie eine Komplettläsion versorgen. Hier wird zunächst die In-situ-Naht beschrieben.

Der Vorteil der In-situ-Naht besteht im Belassen des noch intakten Sehnengewebes am lateralen Tuberculum majus. Hierdurch ist eine Art Schienung der rekonstruierten Anteile gewährleistet und es wird behauptet, dass gleichzeitig weniger Zugbelastung auf das rekonstruierte Gewebe eingeleitet wird.

Nach Überprüfung der Indikation erfolgt zunächst das weitere Vorgehen im Subakromialraum. Je nach intraoperativem Befund führt man die subakromiale Dekompression durch. Auf jeden Fall muss die Sehnenoberfläche freigelegt werden, damit später die vorgelegten Fäden auch wiederum im Subakromialraum gefunden werden können.

Nun erneutes Eingehen in das Glenohumeralgelenk. Die SSP-Unterfläche wird dargestellt. Mit einer Spinalnadel wird die Richtung der transtendinösen Inzision bestimmt. Durch das anterolaterale Portal wird mit einem Skalpell eine longitudinale Stichinzision im Faserverlauf im Bereich der Partialschädigung vorgenommen. Die Inzision kann mit einer Schere oder einem Klemmchen leicht aufgedehnt werden, um so eine stets sicheren Zugang zum Gelenk hat. Man debridiert die Sehne und frischt gleichzeitig die knöcherne Insertion am Tuberculum majus an, sodass punktuelle Blutungen entstehen. Diese dienen der fibroblastischen Einheilung der Sehne. Von lateral wird ein doppelt armierter Fadenanker am osteochondralen Übergang ins Tuberculum majus eingedreht.

Ein Fadenende bringt man mit der Fadenfasszange von lateral ins Gelenk und legt ihn vor das anteroinferiore Portal. Mit einem SutureLasso (Fa. Arthrex, Karlsfeld) perforiert man nun die SSPSehne von lateral und durchsticht die Sehne kranial des retrahierten artikularseitigen Sehnenanteils, den es gilt zu reponieren und zu refixieren. Von anteroinferior wird dann der Lassofaden und der dort geparkte Faden nach außen gezogen, das Fadenende ins Lasso eingelegt und mit dem Lassogriff wieder zusammen nach lateral herausgezogen. So ist ein Fadenende durch die Sehne vorgelegt. Man wiederholt diesen Schritt 2-mal, zunächst mit einem andersfarbigen Faden und dann wieder mit dem anderen Ende des 1. Fadens. Der 2. Stich sollt dabei etwas weiter medial liegen, sodass eine Mason-Allen-Konfiguration entsteht.

Das Arthroskop wechselt nun wieder in den Subakromialraum und man sucht die Fäden auf und verknotet die Fäden, zuerst die vorgelegte horizontale Matratzennaht und letztlich die vertikale Einzelknopfnaht (Abb.4).

Alternativ kann man auch alle 4 Fadenenden durch die Sehne vorlegen und so 2 horizontale Matratzennähte kreieren. Die Fadenenden können dann im Sinne einer Double Row mittels knotenfreier Anker am lateralen Kortex verankert werden.

Entfernen des Instrumentariums, Hautnähte, Kompressionsverband und Anlage einer Gilchrist-Bandage schließen den Eingriff ab.

\section{Tipps und Tricks}

Das anterolaterale Portal sollte nicht zu tief angelegt werden, da sonst der Angriffswinkel zum Einbringen des Nahtankers zu flach werden kann und der Anker sehr tangential mit der Gefahr der Knorpelperforation eingebracht wird.

Die Perforation der Sehne muss möglichst in kraniokaudaler Richtung vorgenommen werden, um nicht $\mathrm{zu}$ weit medial im Gelenk herauszukommen.

Nach Castagna (persönliche Kommunikation) liegt das Limit dieser Technik bei einer Retraktion des intraartikulären Sehnenanteils von $8 \mathrm{~mm}$. Andernfalls ist eine Reposition nicht möglich und es muss doch eine Komplettierung des Sehnenrisses erfolgen, damit die Sehne mobilisiert und reponiert werden kann.

Die bursalseitige Supraspinatuspartialruptur ist im Vergleich zur gelenkseitigen wesentlich schmerzhafter und betrifft ein älteres Patientengut. Der akromialseitige Partialeinriss gehört in die Gruppe des Outlet-Impingements und ist durch einen Akromionsporn mechanisch bedingt. Die bursalseitigen Einrisse führen oft durch eine Lappenbildung zu echten mechanischen Blockaden mit Schnapp-Phänomen. Die Einrisse können die ganze Sehnentiefe betreffen, wobei dann nur noch die superiore glenohumerale Kapselmembran stehenbleibt, welche arthroskopisch an der Unterfläche, d.h. artikularseitig eine intakte Supraspinatussehne vortäuscht.

Für oberflächliche bursalseitige Rupturen Grad I nach Ellman genügt die alleinige subakromiale Dekompression und Sehnenglättung. Für tiefere Defekte ist die arthroskopische oder Mini-OpenSehnenrekonstruktion angezeigt. Es hat sich als sinnvoll erwiesen, eine bursalseitige Partialläsion zunächst in eine kleine Komplettruptur zu verwandeln und dann als solche zu rekonstruieren (Technik s.u.).

\section{Subscapularis}

Die Läsionen des Subscapularis sind häufig klinisch okkult und werden nur durch subtile klinische Untersuchung oder gar erst intraoperativ arthroskopisch entdeckt. Da sie stets auch eine Mitbeteiligung des Pulley-Systems aufweisen, führen sie durch die Affektion der langen Bizepssehne zu entsprechenden Symptomen. Heute ist die Versorgung dieser 


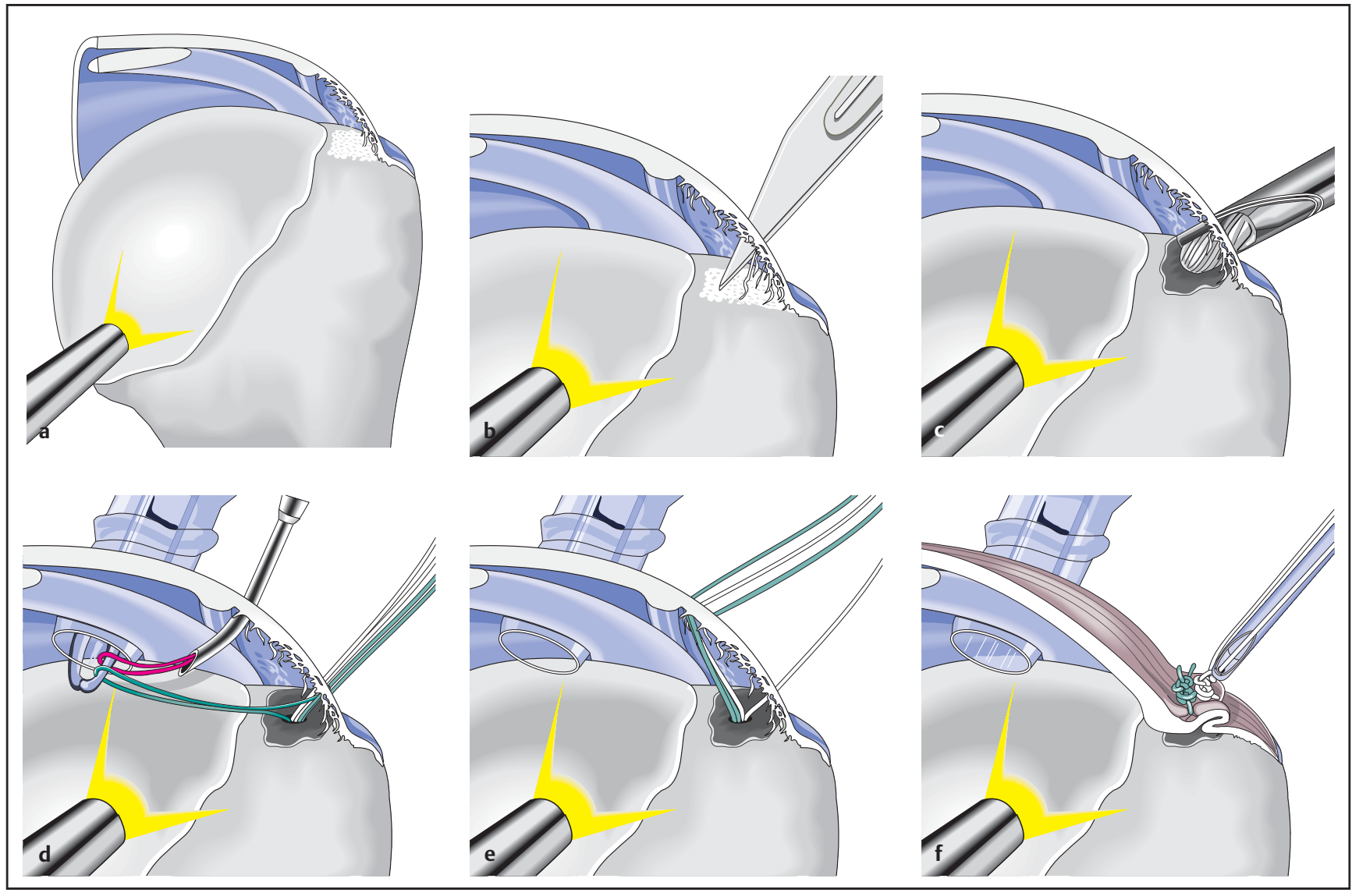

Abb. 4 Rekonstruktion der artikularseitigen Rotatorenmanschettenpartialruptur. a Artikularseitige Supraspinatussehnenpartialruptur mit mehr als $50 \%$ Ausriss der Sehne von ihrem Ansatz (Footprint). b Longitudinale Inzision der Supraspinatussehne im betroffenen Areal von subakromial. c Anfrischen des Footprints, bis einzelne Blutungen auftreten. d Perforation der Läsion mit einem Lasso und gleichzeitiges Herausziehen des Lassofadens (blau) und des Ankerfadens (grün) nach anterior. Hier wird der Ankerfaden in das Lasso eingelegt und nach lateral gezogen. e Die Schritte von Abbildung 4 d werden 2-mal wiederholt, sodass 2 gleichfarbige Fäden und ein andersfarbiger Faden zwischen ihnen durch die Sehne vorgelegt sind. Ein Fadenende bleibt frei. Dies stellt eine modifizierte Mason-Allen-Naht dar. f Nach Verknotung der Fäden ist die Sehne wieder kräftig auf dem Footprint-Areal refixiert (Mit freundlicher Genehmigung aus: Lichtenberg, Habermeyer, Magosch. Atlas Schulterarthroskopie. ( E) Elsevier GmbH).

Läsionen eher aggressiver, was bedeutet, dass diese Schädigungen rekonstruiert werden sollten. Die als störendes Agens vorhandene Bizepssehne wird entweder tenotomiert oder mit einer Tenodese versorgt.

Die Rekonstruktion der Subscapularissehnen erfolgt arthroskopisch.

Nach der diagnostischen Arthroskopie, die der Überprüfung der Indikation dient, beginnt man nach Anlage des anteroinferioren Portals mit dem Anfrischen des Ansatzgebiets. Morphologisch stellt sich die Läsion so dar, dass der obere Anteil der Sehne mit oder ohne den humeralen Ansatz des superioren glenohumeralen Ligaments (SGHL) von der knöchernen Insertion abgelöst ist. Nach ausreichendem Anfrischen des Knochens, bis punktuelle Blutungen entstanden sind, wird ein doppelt armierter Nahtanker eingebracht.
Subakromial muss zunächst ein ausreichendes Débridement stattfinden. Dann wird eine kleine Stichinzision in die Supraspinatussehne vorgenommen. Nun wird von ventral durch das anteroinferiore Portal die Subscapularissehne mit einem SutureLasso perforiert und der Lassofaden nach intraartikulär eingeleitet. Von lateral Einführen einer Fadenfasszange, mit der gleichzeitig der Lassofaden und ein Ankerfaden nach lateral herausgezogen wird. Der Faden wird ins Lasso eingelegt und nun nach ventral gezogen. Diese Schrittfolge wird insgesamt 2-mal wiederholt, sodass eine modifizierte Mason-Allen-Naht durch die Subscapularissehne vorgelegt wurde. Bei Mitbeteiligung des SGHL kann nun zusätzlich dieses perforiert werden und so auch dieses in die Naht integriert werden.

Man überprüft die Lage der Fäden und unter Probezug sollte sich die Sehne ins Sehnenlager reponieren. Dann verknotet man zunächst den horizontalen Matratzenfaden und dann den vertikalen Faden (Abb. 5).

Bei Mitbeteiligung des medialen LBSPulleys und bereits eingetretener Schädigung der langen Bizepssehne ist diese mit einer Tenodese zu versorgen.

\section{Tipps und Tricks}

Das Erreichen des oberen Anteils des Tuberculum minus kann manchmal schwierig sein. Der OP-Assistent muss dann den Arm mehr flektieren und verschiedene Rotationsstellungen probieren, bis man optimale Sicht hat. Auch das Einbringen des Ankers ist aus gleichem Grund erschwert. Hier bietet es sich an, das anteriore Portal etwas weiter lateral anzulegen, sodass man direkt über der Bizepssehne das Intervall inzidiert. Dies erleichtert auch die Platzierung der Fäden zur Bizepssehnen-Tenodese. 


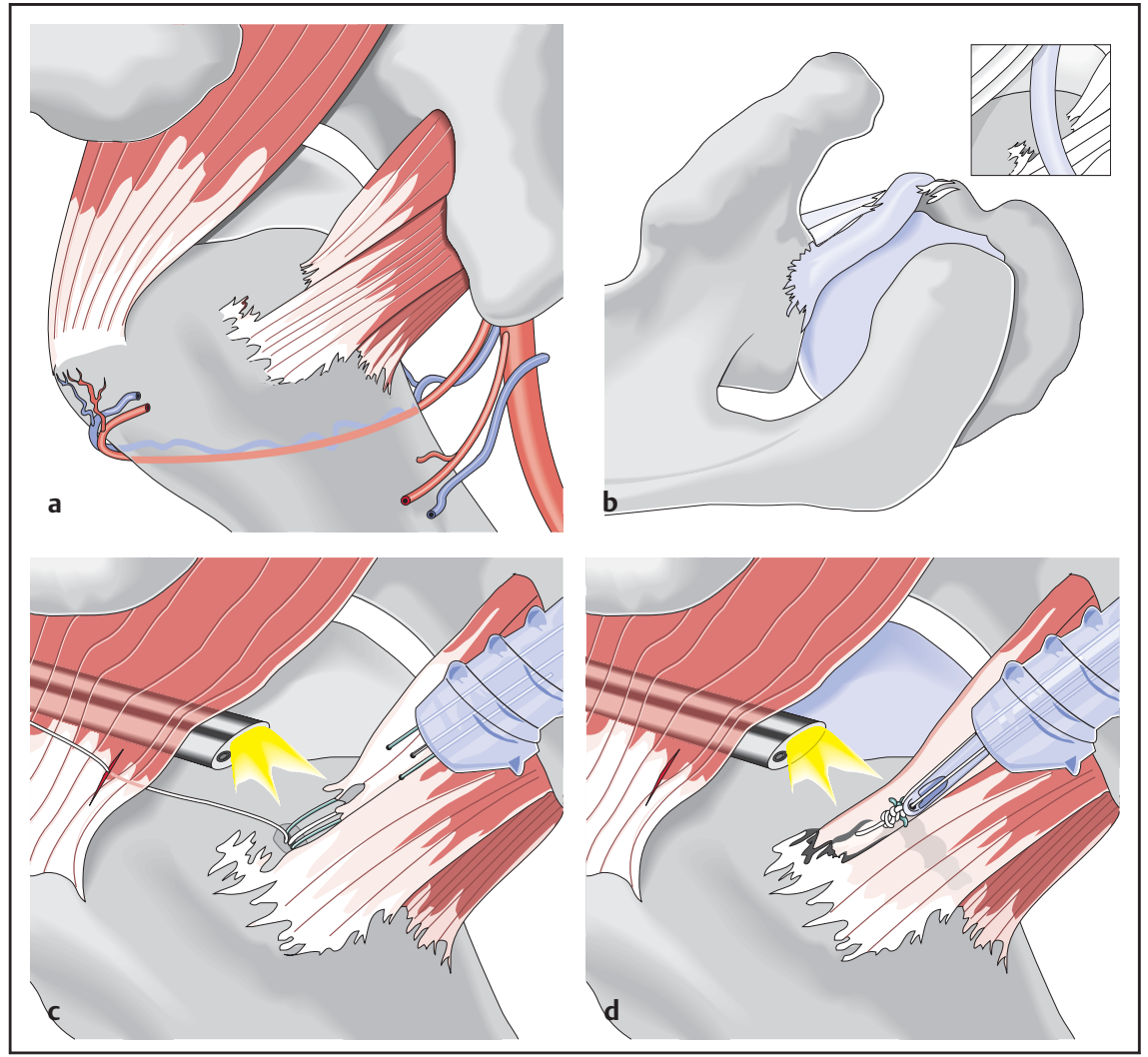

Abb.5 Arthroskopische Rekonstruktion der Subscapularissehnenpartialläsion. a Subscapularissehnenpartialläsion mit teilweisem Ausriss der Sehne aus dem oberen Bereich des Tuberculum minus. b Bei dieser Verletzung kommt es häufig zur Mitverletzung des medialen Pulley-Systems mit konsekutiver Sub- oder Luxation der langen Bizepssehne. c Nach Anfrischen des Footprints und Implantation eines Nahtankers erfolgt die Perforation der Sehne von anterior mit dem Lasso. Von lateral durch eine kleine Stichinzision im M. supraspinatus werden der Ankerfaden und der Lassofaden herausgezogen. Dieser Schritt wird 2-mal wiederholt, bis eine modifizierte Mason-Allen-Naht entstanden ist. d Rekonstruierte Subscapularissehne (Mit freundlicher Genehmigung aus: Lichtenberg, Habermeyer, Magosch. Atlas Schulterarthroskopie. ( Elsevier GmbH).

\section{Rekonstruktion von kompletten und kombinierten Sehenrupturen}

Auch solche Verfahren sind heute allesamt technisch arthroskopisch machbar. Die Indikationsstellung entscheidet über den Erfolg der OP. Das soll nicht bedeuten, dass man weniger Läsionen zur Rekonstruktion indiziert, sondern dass man eher vorsichtig ist und nicht jedem Patienten eine Sehnenrekonstruktion in Aussicht stellt, obwohl objektiv keine reelle Rekonstruktionschance mehr besteht.

Die Differenzialindikation zwischen irreparabel, reparabel und teilreparabel mittels Muskeltransfer ist die schwierigste Aufgabe in der Chirurgie der Sehnenläsionen der Schulter.

Die Grundprinzipien der Sehnenrekonstruktion bleiben auch beim arthroskopischen Vorgehen die gleichen:
Eine Sehne, die nur am knöchernen Ansatz am Tuberculum majus reißt, retrahiert von lateral nach medial, wobei je nach Ausdehnung der Läsion am Knochen sich die Sehne entzweit und große U-förmige Rupturen entstehen lässt.

Eine Sehne, die aber eine zusätzliche longitudinale Läsion entlang des Rotatorenintervalls aufweist, zieht sich eher nach posterolateral zurück und umgekehrt retrahiert die Sehne nach anteromedial, wenn eine zusätzliche dorsale longitudinale Läsion vorliegt.

\section{M. subscapularis}

Abgesehen von der isolierten traumatischen Ruptur des Subscapularis beim jüngeren Patienten nach adäquatem Trauma tritt die Läsion des Subscapularis in Kombination mit Supraspinatusrupturen auf.

Wenn nur der obere intraartikuläre Anteil der Sehne geschädigt ist, wird die Sehne schon bei der diagnostischen Arthroskopie über das dorsale Portal sichtbar. Bei Kombination mit einer großen superioren Manschettenruptur kann die Sehne jedoch schon bis auf Glenoidniveau oder auch medial davon retrahiert sein. Dann kann die Sehne erst gesehen werden, nachdem man mit einer Fasszange von lateral kommend das $\mathrm{Ge}$ webekonglomerat fasst und nach lateral zieht. Es kommt dann die typische Anatomie von oberem Subscapularisrand, Rotatorenintervallkapsel und Resten des superioren glenohumeralen Bandes (SGHL) zum Vorschein. Dies ist das von Burkhart beschriebene Comma Sign [11]. Die Subscapularissehne ist also in Kontinuität mit dem Supraspinatus von der knöchernen Insertion ausgerissen und retrahiert. Es empfiehlt sich dann, einen Haltefaden sowohl in die Subscapulariswie auch die Supraspinatussehne zur weiteren Mobilisation vorzulegen. Lässt sich die Sehne des Subscapularis mit der Zange oder am Haltefaden gut nach lateral ziehen, ist die Mobilisation nicht schwierig. Bei sehr steifer Sehne muss dann ein entsprechendes Release der Kapsel und des SGHL-Ansatzes am Glenoid auf der Rückseite der Sehne erfolgen. Später schließt sich die subkorakoidale Mobilisation mit Durchtrennung des CHL und Bridenlösung zum Korakoid hin an. Bei sehr steifen kontrakten Verhältnissen ist der anteriore IntervallSlide durchzuführen.

- arthroskopische Technik der isolierten SSP-Rekonstruktion 


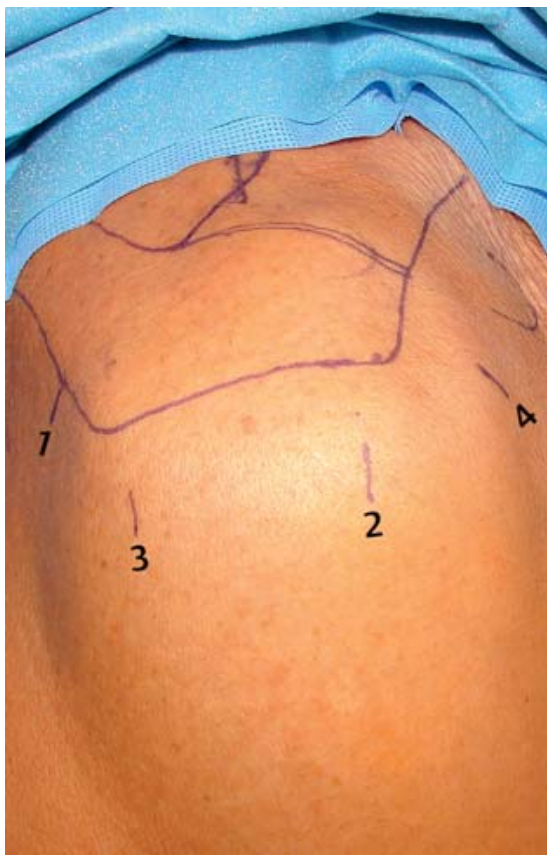

Abb. 6 Portale für die arthroskopische Supraspinatussehnenrekonstruktion. 1: dorsales Standardportal; 2: anterolaterales subakromiales Portal; 3: posterolaterales subakromiales Portal; 4: anterosuperiores subakromiales Portal.

- Lagerung

- Beach-Chair Lagerung

- Portale (Abb. 6)

- dorsales Standardportal

- anterolaterales subakromiales Portal

- posterolaterales subakromiales Portal

- anterosuperiores subakromiales Portal

\section{OP-Technik}

Ziel der Operation ist es, die komplett abgelöste Sehne wieder in ihrem Insertionsgebiet (Footprint) zu befestigen.

Während der diagnostischen Arthroskopie erfolgt die Größenbestimmung des Risses von intraartikulär wie auch von subakromial.

Alle Begleitverletzungen, wie z.B. Bizepssehnensubluxation oder -partialschädigung, und Begleitveränderungen wie Impingement und/oder AC-Arthrose werden zu Beginn der Operation behandelt.

Ist dies geschehen, wird mit dem Arthroskop im lateralen subakromialen Portal und dem Shaver von posterior zunächst ein ausführliches Débridement der SSPOberfläche durchgeführt. Die Sicht behindernden Anteile der Bursa werden mit dem Shaver und einer Elektrosonde entfernt, bis die gesamte SSP gut eingesehen werden kann. Nun wird in Outside-in-Technik ein posterolaterales Portal angelegt. Die Spinalnadel sollte ungefähr in der Mitte zwischen anterolateralem und posteriorem Portal liegen und von der Richtung so eingebracht werden, dass die Nadel in die Ruptur zeigt. Dann Umstecken der Optik nach posterolateral. Mit dem Shaver entfernt man die Sehnenreste auf dem Tuberculum majus. Mit einer Gewebefasszange kann nun eine Probereposition erfolgen. Hierdurch kann die richtige Zug- und Rekonstruktionsrichtung bestimmt werden. Ferner legt man so fest, wie weit und wo die Sehne mobilisiert werden muss. Dann kreiert man mit der Walzenfräse frische Blutungen auf dem Tuberculum majus zur fibroblastischen Einheilung der Sehne.

\section{Single-Row-Repair}

Hierzu wird ein Nahtanker, der mit 2 Fäden armiert ist, ins Tuberculum majus implantiert. Dies erfolgt durch ein zusätzliches anterosuperiores Portal, das so angebracht wird, dass man einen guten Aktionswinkel auf das Tuberculum hat. Die Stelle sollte mittig zwischen osteochondralem Übergang und lateralem Rand liegen, mehr mit der Tendenz nach lateral. Je nach Rissbreite sind auch 2 Anker notwendig. Pro $1 \mathrm{~cm}$ Rissbreite sollte ein Anker verwendet werden.

\section{Nahtzange}

Nun wird ein Fadenende lateral heraus gezogen und in die Nahtzange eingelegt. Die Nahtzange wird von lateral eingeführt. Die Brachen greifen tief in die Sehne bis zum Anschlag der Zange, dann wird durch Zusammenkneifen der Zange der Faden durch die Sehne transportiert und über das anterosuperiore Portal mit einer Fasszange geborgen. Dieser Schritt wird so wiederholt, dass der 2. Stich medial und dorsal des 1 . und der 3. dorsal und lateral des 2. liegt. Es resultiert eine modifizierte Mason-Allen-Konfiguration. Bei Verwendung von 2 Ankern werden die Fäden des 1 . Ankers mit einer Klemme gesichert und der 2 . Anker ca. $1 \mathrm{~cm}$ weiter dorsal platziert. Die Schritte für die Sehnenperforation werden analog wiederholt. Das Erreichen der dorsalen Strukturen erleichtert man sich, indem der Assistent den Arm entsprechend abduziert und innen rotiert.

Die Fäden werden dann sukzessiv von dorsal nach ventral verknotet, wobei zu- nächst die horizontale, dann die vertikale Naht verknotet wird.

\section{Lasso-Naht (oder andere Perforationsinstrumente)}

Gerade bei der isolierten Ruptur ist es wichtig, dass das kräftige gesunde Gewebe der Sehne in das vorbereitete Sehnenlager fixiert wird. Dies erkennt man am besten von intraartikulär. Deshalb versorgt man diese Ruptur bei Verwendung eines Perforationsinstruments mit der Optik im dorsalen intraartikulärem Portal.

Nach Einbringen des Nahtankers wird mit dem SutureLasso (Fa. Arthrex, Karlsfeld) über das anterosuperiore Portal die Sehne medial des Fasciculus obliquus (Rotatorenkabel) durchstochen. Nun nimmt man mit einer Fasszange von lateral kommend den Lasso- und den entsprechenden Ankerfaden gleichzeitig auf und führt sie nach lateral heraus. Der Faden wird ins Lasso eingelegt und retrograd nach anterosuperior zurückgezogen. Dieser Schritt wird analog zum oben erwähnten 2-mal wiederholt, bis eine modifizierte Mason-Allen-Naht entstanden ist. Ist ein 2. Anker notwendig, wird dieser wieder über das anterosuperiore Portal platziert. Zur Versorgung der Sehne ist nun aber die Optik im Weg, weshalb sie über das anterolaterale Portal eingeführt wird. Man hat nun einen schönen Überblick auch über die posterioren Anteile der Ruptur. Mit dem Lasso wird nun die Sehne von dorsal kommend perforiert und der Lasso- und der Ankerfaden über das posterolaterale Portal herausgezogen und versorgt. Nach Vorlegen der Mason-Allen-Naht liegen die 3 durch die Sehne geführten Fäden im dorsalen Portal. Von hier sollten sie nach anterosuperior umgelagert werden. Dann Umstecken der Optik nach posterolateral und sukzessives Verknoten der vorgelegten Fäden von dorsal nach ventral. Abschließend erfolgt eine Überprüfung der Rekonstruktion auch von intraartikulär.

\section{Double-Row-Rekonstruktion}

Der Vorteil der doppelten Ankerreihe besteht in einem flächigeren Kontakt der Sehne in das Tuberculum majus.

Zunächst wird ein 1. Anker am osteochondralen Übergang eingebracht und mit der Optik im dorsalen intraartikulären Portal mittels der Lasso-Technik die Sehne versorgt. Hierbei werden nun aber 
2 horizontal Matratzennähte vorgelegt, da eine Mason-Allen-Naht das weitere Lateralisieren der Sehne verhindern würde. Bei Bedarf wird ein 2. Anker weiter dorsal am osteochondralen Übergang eingebracht und nach Umstecken der Optik ebenfalls 2 Matratzennähte vorgelegt. Um nicht zu viele Perforationsstellen zu haben, können beim 2. Perforieren 2 Fäden mit dem Lassofaden durch die Sehne gezogen werden. Hier ist aber darauf zu achten, dass dies 2 unterschiedliche Fadenenden sind. Nun sind insgesamt 4 horizontale Nähte vorgelegt. Würde man dies verknoten, käme es zu einem Verschluss der Sehne am osteochondralen Übergang. Dies macht die Rekonstruktion primär wasserdicht, hinterlässt jedoch einen lateralen Sehnenanteil, der sich bei jeder Bewegung vom lateralen Footprint-Areal abheben würde. Hierzu werden nun auch lateral weitere Anker eingebracht.

\section{Laterale Reihe mit weiteren Nahtankern}

Je nach Breitenausdehnung kann 1 Anker oder müssen 2 Anker eingebracht werden.

Wird nur 1 Anker verwendet, muss dieser mittig zwischen den beiden medialen, aber möglichst lateral am Kortex eingebracht werden. Mit dem SutureLasso werden dann 2 vertikale Einzelknopfnähte vorgelegt. Hierdurch wird nun das laterale Sehnengewebe kräftig in das Tuberculum majus fixiert. Bei 2 lateralen Ankern werden analog 4 vertikale Einzelknopfnähte vorgelegt.

Prinzipiell ist es egal welche Nahtreihe man zuerst verknotet, aber es kann zu Schwierigkeiten folgender Art kommen:

Knotet man zunächst medial, kann es vorkommen, dass nun lateral nicht genügend Gewebe zum Decken des Footprints vorhanden ist, da die medialen Fäden letztlich doch $\mathrm{zu}$ weit lateral durch die Sehne vorgelegt wurden und nun das Gewebe nach medial gezogen wird.

Knotet man zunächst lateral, kann es passieren, dass nun medial Luftknoten entstehen und die medialen Sehnenanteile nicht sicher am osteochondralen Übergang am Knochen fixiert werden. Deshalb ist es wichtig, die Fäden perfekt zu platzieren.

Manchmal muss man unter Probezug an den vorgelegten Fäden entscheiden, was die bessere Alternative ist und wie sich das Sehnengewebe verhält.

Nach ausführlicher Spülung erfolgen der Hautverschluss und die Installation von Lokalanästhetikum. Anlage einer Gilchrist-Bandage.

Laterale Reihe mit knotenfreiem Anker (SutureBridge-Technik; transosseous equivalent-Technik)

Alternativ zu einem konventionellen Anker kann auch ein knotenfreier Anker für die laterale Fixierung verwendet werden.

Hierzu verwendet man die zuvor geknoteten Fäden der medialen Reihe. Bei Verwendung eines medialen Ankers existieren 2 mediale Knoten mit 4 Fadenenden. Die Fäden werden im anterosuperioren Portal geparkt. Man zieht nun je 1 Fadenende eines jeden Knotens über das anterolaterale Portal heraus. Ein Aufnahmeloch für einen knotenfreien Anker (PushLock, Fa. Arthrex, Karlsfeld) wird am ventralen Rand der Ruptur am lateralen Cortex humeri vorgekörnt. Die Fäden werden in den PushLock-Applikator eingebracht und dieser durch die gleiche Inzision an den Pfriem herangebracht. Man muss dieses Loch sicher wiederfinden, da sonst ein Einbringen des Ankers nicht gelingt. Ferner muss in der gleichen Richtung, wie der Pfriem eingetrieben wurde, auch der Applikator ausgerichtet sein, da sonst der Anker nicht ins Loch eingebracht werden kann, bzw. das Öhr auf dem Applikator verrutscht und keine suffiziente Verankerung der Fäden erfolgt. Die Industrie wartet hier mit einer Modifikation des Ankers auf, nämlich besteht das Öhr, in das die Fäden eingelegt werden, aus Titan und ist angespitzt, sodass der Schritt des Vorkörnens entfällt. Nachdem die Fäden mit dem Anker sicher fixiert sind, schneidet man die überstehenden Fadenreste ab. Dann zieht man die übrigen beiden Fadenenden nach lateral und versorgt diese mit einem zweiten PushLock-Anker. Dieser wird ebenfalls am lateralen Cortex humeri eingebracht und zwar am dorsalen Rupturrand. Es resultiert eine gekreuzte Fadenkonfiguration, durch die der laterale Sehnenanteil nun schön und kräftig im lateralen Insertionsgebiet am Tuberculum majus fixiert wurde.

Hat man medial 2 Anker mit 4 Knoten platziert, verwendet man für den ventralen PushLock jeweils die Fäden der ventralen Knoten eines jeden Anker und für den dorsalen PushLock jeweils die Fäden der dorsalen Knoten eines jeden Ankers (Abb. 7).

Die Ergebnisse der arthroskopischen Rotatorenmanschettenrekonstruktion sind vergleichbar mit denen der offenen und „mini-open“-Verfahren. Heutzutage muss sich die arthroskopische Technik nicht mehr hinter den offenen Verfahren verstecken. Die Ergebnisse sind in den Tab. 3-6 dargestellt.

Gewisse Faktoren können aus den Ergebnissen in der Literatur herausgearbeitet werden.

\section{Einflussfaktoren auf das postoperative Ergebnis}

\section{Alter}

Sowohl die Arbeit von Lichtenberg et al. [8] als auch die von Boileau et al. [12] zeigten eine höhere Re-Ruptur-Häufigkeit bei Patienten über 65 Jahren. Cole et al. [13] konnten bei über 70-jährigen eine Re-Ruptur-Rate von 62,5\% ermitteln.

\section{Atrophie und fettige Infiltration}

Die präoperative Atrophie und fettige Degeneration der betroffenen Sehnen waren negative Einflussfaktoren auf die postoperative Sehnenintegrität. Zum einen zeigte eine Arbeit von Liem et al. [14], dass bei einer Atrophie Stadium I weniger Re-Rupturen auftraten als bei Patienten, die schon präoperativ eine Atrophie Stadium II besaßen. Dies wird auch hinsichtlich der fettigen Atrophie bestätigt, insofern als bei fettiger Infiltration Grad II präoperativ mehr Re-Rupturen $\mathrm{zu}$ verzeichnen waren als bei Muskeln ohne eine präoperative fettige Infiltration.

\section{Rissgröße}

In unserer Arbeit [8] zeigte sich statistisch nur ein Trend zu mehr Re-Rupturen, je größer der Riss intraoperativ gemessen wurde. Boileau et al. [12] sahen eine schlechtere Einheilungsquote, wenn die Ruptur zusätzlich eine Delamination in den Infraspinatus aufwies (Re-RupturRate $51 \%$ ). Auch Cole bestätigt dies mit einer Re-Ruptur-Rate von $46 \%$ bei Mitbeteiligung des Infraspinatus. Höhere Re-Ruptur-Raten waren bei Bishop et al. sowohl in der mini-open als auch in der arthroskopisch operierten Gruppe zu beobachten. 


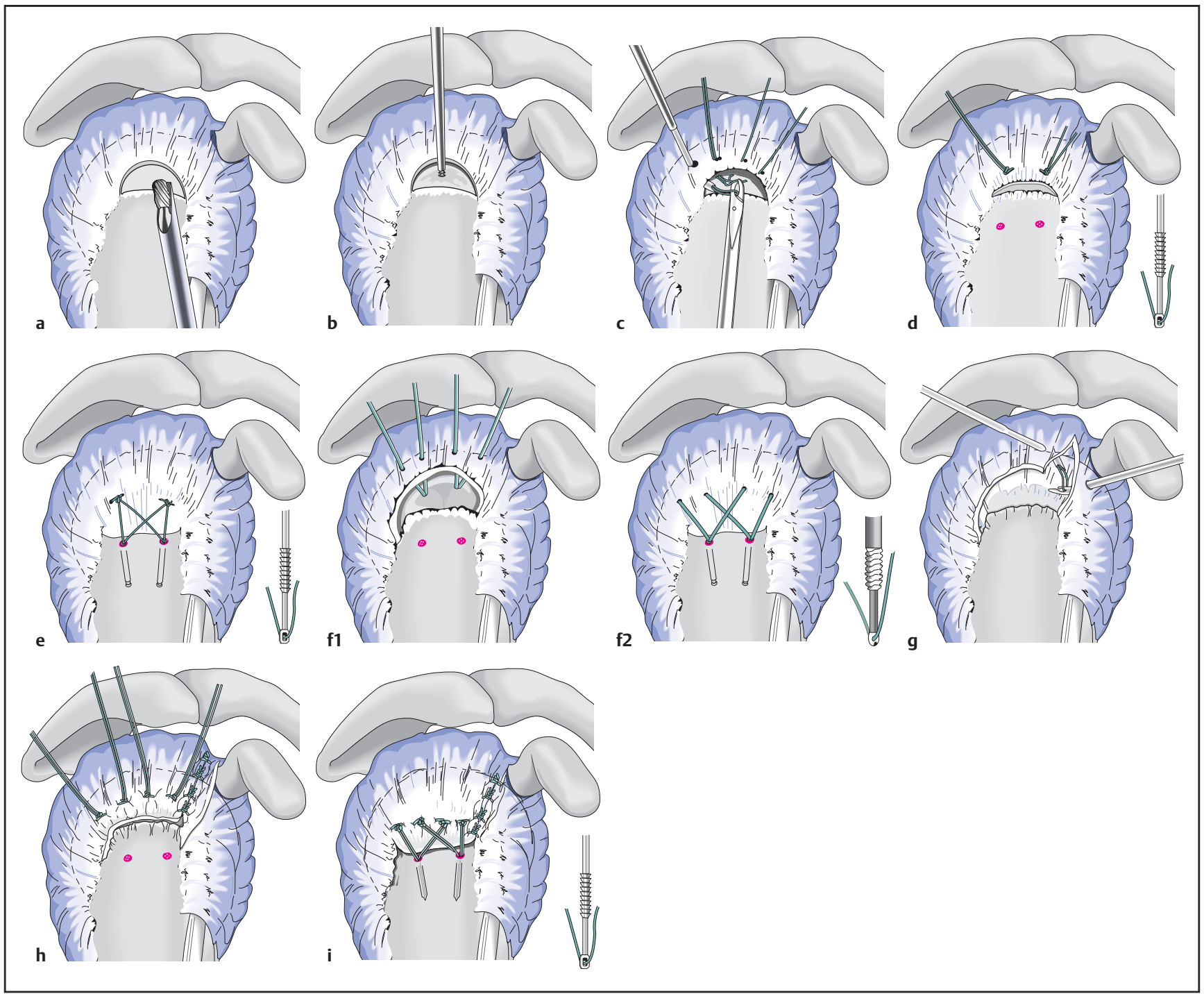

Abb. 7 Arthroskopische Rotatorenmanschettenrekonstruktion in Double-Row-Technik. a Anfrischen des Sehnenlagers mit einer Fräse. Hier wird bis zum Auftreten von Blutungen gefräst, ohne in den spongiösen Knochen zu geraten. Dies könnte zu einer Schwächung des Knochens mit der Gefahr des Fadenankerausrisses führen. b Einbringen eines Nahtankers mit 2 Fäden. c Mit einem Perforationsinstrument werden alle 4 Fadenenden durch die Supraspinatussehne vorgelegt, sodass 2 horizontale Matratzennähte entstehen. $\mathbf{d}$ Die Fäden sind im Sinne von Matratzennähten verknotet. Die Implantationsorte für die laterale Reihe sind rot markiert. e Die laterale Reihe an Ankern wird mit knotenfreien Ankern vorgenommen. Jeweils ein Faden eines jeden Knotens wird in einen PushLock-Anker eingebracht und am lateralen Kortex fixiert. $\mathbf{f}$ Bei Verwendung der SpeedBridge-Technik kommt der Operateur völlig ohne Knoten aus. Die beiden Fiber-Tape-Enden eines jeden Ankers werden durch die Supraspinatussehne perforiert und dann gekreuzt über 2 Anker am lateralen Kortex im Knochen versenkt. g Versorgung einer anterioren (reversen) L-förmigen Ruptur. Zunächst Vorlegen von Seit-zu-Seit-Fäden mit Lasso und BirdBeak. h Anschließend Setzen der Nahtanker medial und Vorlegen mehrerer Matratzennähte. i Abschließendes Verknoten und Einbringen der lateralen knotenfreien Anker (Mit freundlicher Genehmigung aus: Habermeyer, Lichtenberg, Magosch. Schulterchirurgie. (c) Elsevier GmbH).

Setzt man die Rissgröße in Relation zum klinischen Ergebnis, fällt in den Arbeiten von Wilson et al. [15] und von Lee et al. [16] ein schlechteres Score-Ergebnis auf. Wilson zeigte auch, dass die Außenrotation bei Rissen über $3 \mathrm{~cm}$ Breite sowohl prä- wie auch postoperativ signifikant schlechter war. Einzig Burkhart [17] sieht keinen Einfluss der Rissgröße auf das postoperative Ergebnis.

\section{Faktor Re-Ruptur}

In allen zitierten Arbeiten fällt eine klare Tendenz auf, dass die Patienten mit einer Re-Ruptur schlechtere Score-Werte besaßen. Dies ist v.a. der Tatsache geschuldet, dass bei den Scores die Kraftwerte einen hohen Anteil am Gesamtergebnis ausmachen. Insbesondere die Außenrotationskraft ist bei Patienten mit ReRuptur in der Regel geringer, weniger stark ist dieser Unterschied bei der anterioren Elevation ausgeprägt. Alle Patien- ten haben aber unabhängig vom postoperativen Sehnenstatus signifikant verbesserte Werte für Schmerz, Aktivitäten des täglichen Lebens und Bewegungsumfänge. Die Patienten zeigen sich durch alle Arbeiten hindurch mit dem postoperativen Ergebnis zufrieden.

Cole et al. [13] konnten auch zeigen, dass sich die Patienten auch noch zwischen der 1-Jahres- und der 2-Jahres-Kontrolle verbessern, sofern die Sehnen intakt waren. Galatz [18] dagegen untersuchte die 
Tab. 3 Ergebnisse der arthroskopischen Rekonstruktion kleiner bis mittelgroßer Rotatorenmanschettenrupturen.

\begin{tabular}{llllll} 
Autor & $\begin{array}{l}\text { Anzahl der } \\
\text { Patienten }\end{array}$ & $\begin{array}{l}\text { Nachuntersu- } \\
\text { chungszeitraum }\end{array}$ & Rupturgröße & präoperativ & postoperativ \\
\hline $\begin{array}{l}\text { Bennett } \\
\text { Arthroscopy 2003 }\end{array}$ & 37 & 2-4 Jahre & klein-mittel & 54 CS & 73 \\
\hline $\begin{array}{l}\text { Wilson et al. } \\
\text { Arthoscopy 2002 }\end{array}$ & 30 & $2-14$ Jahre & $<2$ & 21 UCLA & 33,5 \\
\hline $\begin{array}{l}\text { Murray et al. } \\
\text { JSES 2002 }\end{array}$ & 42 & 39 Monate & $2,4 \mathrm{~cm}$ & 17,2 UCLA & 33,7 \\
\hline $\begin{array}{l}\text { Lee et al. } \\
\text { JSES 2007 }\end{array}$ & 36 & $(24-66)$ & 42,2 ASES & 94,9 \\
\hline
\end{tabular}

Tab. 4 Ergebnisse der arthroskopischen Rekonstruktion großer und Massenrupturen der Rotatorenmanschette.

\begin{tabular}{|c|c|c|c|c|c|}
\hline Autor & Patientenanzahl & $\begin{array}{l}\text { Nachuntersu- } \\
\text { chungszeitraum }\end{array}$ & Rupturgröße & präoperativ & postoperativ \\
\hline $\begin{array}{l}\text { Bennett } \\
\text { Arthrocopy } 2003\end{array}$ & 37 & 2-4 Jahre & massiv & $47 \mathrm{CS}$ & 74 \\
\hline $\begin{array}{l}\text { Wilson et al. } \\
\text { Arthroscopy } 2002\end{array}$ & 3 & 2-14 Jahre & $>4 \mathrm{~cm}$ & ?UCLA & 24,5 \\
\hline $\begin{array}{l}\text { Lee et al. } \\
\text { JSES } 2007\end{array}$ & $\begin{array}{l}14 \\
21\end{array}$ & $\begin{array}{l}\text { 16,5 Monate } \\
(12-45)\end{array}$ & $\begin{array}{l}>3 \mathrm{~cm} \\
>5 \mathrm{~cm}\end{array}$ & $\begin{array}{l}43 \text { ASES } \\
50 \text { CS } \\
41 \text { ASES } \\
50 C S\end{array}$ & $\begin{array}{l}74 \\
74 \\
67 \\
68\end{array}$ \\
\hline $\begin{array}{l}\text { Burkhart et al. } \\
2007\end{array}$ & 22 & $\begin{array}{l}\text { 39,3 Monate } \\
(24-60)\end{array}$ & massiv & 12,3 UCLA & $\begin{array}{l}29,5 \\
88,5 \% \text { CS }\end{array}$ \\
\hline
\end{tabular}

Tab. 5 Ergebnisse nach arthrokopischer Versorgung von Rotatorenmanschettenrupturen mit bildgebender Kontrolle.

\begin{tabular}{|c|c|c|c|c|c|c|c|}
\hline Autor & Patientenanzahl & Bildgebung & FU Monate & Größe & Re-Ruptur & präoperativ & $\begin{array}{l}\text { post- } \\
\text { operativ }\end{array}$ \\
\hline $\begin{array}{l}\text { Lichtenberg et al. } \\
\text { KSSTA } 2006\end{array}$ & 53 & MRI & $26,5(\min 24)$ & SSP & $25 \%$ & $53 \mathrm{CS}$ & 83 \\
\hline $\begin{array}{l}\text { Boileau et al. } \\
\text { JBJS A } 2005\end{array}$ & 51 & $\mathrm{MRI} / \mathrm{CT}$ & 29 & $\begin{array}{l}\text { SSP } \pm \text { Delami- } \\
\text { nation ISP }\end{array}$ & $28 \%$ & $51,6 \mathrm{CS}$ & 83,8 \\
\hline $\begin{array}{l}\text { Cole et al. } \\
\text { JSES } 2007\end{array}$ & 49 & MRI & $32(24-45)$ & $\begin{array}{l}\text { klein - Mas- } \\
\text { senruptur }\end{array}$ & $22 \%$ & $\begin{array}{l}43 \text { ASES } \\
49 \text { CS }\end{array}$ & $\begin{array}{l}85 \\
76\end{array}$ \\
\hline $\begin{array}{l}\text { Galatz et al. } \\
\text { JBJS A } 2004\end{array}$ & 18 & US & $\begin{array}{l}12 \\
24\end{array}$ & $>2 \mathrm{~cm}$ & $17 / 18$ & 48,3 ASES & $\begin{array}{l}84,61 \\
79,92\end{array}$ \\
\hline $\begin{array}{l}\text { Anderson et al. } \\
\text { AJSM } 2006\end{array}$ & 52 & US & $30(\min 24)$ & $1-4 \mathrm{~cm}$ & $17 \%$ & 42 L'Inslata & 93 \\
\hline
\end{tabular}

Patienten nach 2 Jahren erneut und stellte eine Verschlechterung fest, was aber daran lag, dass es sich um 17 Re-Rupturen von 18 Patienten handelte.

\section{Alternativverfahren bei irreparablen Rupturen}

Zur funktionellen Rekonstruktion der Rotatorenmanschette bieten sich prinzipiell 2 Verfahren an.

- Verlust der anterosuperioren RM = Pectoralis-major-Transfer
- Verlust der posterosuperioren RM = Latissimus-dorsi- oder Teres-majorTransfer

Je nach Verlust der RM können oben genannte Ersatzverfahren gewählt werden. Während der Ersatz für die posterosuperiore RM mittels Latissimus-dorsi- oder Teres-major-Transfer vor allem funktionelle und schmerzpalliative Aspekte hat, besitzt der Pectoralis-major-Transfer meist nur eine schmerzlindernde Funktion.
Indikation zum posterioren Muskelersatz:

- irreparable posterosuperiore RMRuptur

- aktive anteriore Elevation $<90^{\circ}$

- aktive Abduktion <90

- Außenrotations-Lag-Sign

- intakter Subscapularis

- intakter M. deltoideus 
Tab. 6 Ergebnisse der arthroskopischen Double-Row-Rekonstruktion von Rotatorenmanschettenrupturen.

\begin{tabular}{|c|c|c|c|c|c|c|c|}
\hline Autor & $\begin{array}{l}\text { Patienten- } \\
\text { anzahl }\end{array}$ & Bildgebung & $\begin{array}{l}\text { Nachuntersu- } \\
\text { chungszeit- } \\
\text { raum (Monate) }\end{array}$ & Rupturgröße & Re-Ruptur & präoperativ & $\begin{array}{l}\text { post- } \\
\text { operativ }\end{array}$ \\
\hline $\begin{array}{l}\text { Lafosse et al. } \\
\text { JBJS A } 2007\end{array}$ & $\begin{array}{l}105 \\
\text { DR }\end{array}$ & $\begin{array}{l}\text { Arthro-MRI/ } \\
\text { CT }\end{array}$ & $\min .24$ & $\begin{array}{l}\text { klein - Mas- } \\
\text { senruptur }\end{array}$ & $11,4 \%$ & $43,2 \mathrm{CS}$ & 80,1 \\
\hline $\begin{array}{l}\text { Sugaya et al. } \\
\text { JBJS A } 2007\end{array}$ & $\begin{array}{l}106 \\
D R\end{array}$ & MRI & 14 & $\begin{array}{l}\text { klein - Mas- } \\
\text { senruptur }\end{array}$ & $\begin{array}{l}17 \% \\
5 \text { (klein) } \\
40 \text { (mass) }\end{array}$ & $\begin{array}{l}42 \text { ASES } \\
14,5 \text { UCLA }\end{array}$ & $\begin{array}{l}94 \\
32,9\end{array}$ \\
\hline $\begin{array}{l}\text { Huijsmans et al. } \\
\text { JBJS A } 2007\end{array}$ & $\begin{array}{l}264 \\
D R\end{array}$ & US & $\min .12$ & $\begin{array}{l}\text { klein/med. } \\
\text { groß } \\
\text { Massenruptur }\end{array}$ & $\begin{array}{l}12 / 7 \% \\
22 \% \\
53 \%\end{array}$ & $54,9 \mathrm{CS}$ & 80 \\
\hline
\end{tabular}

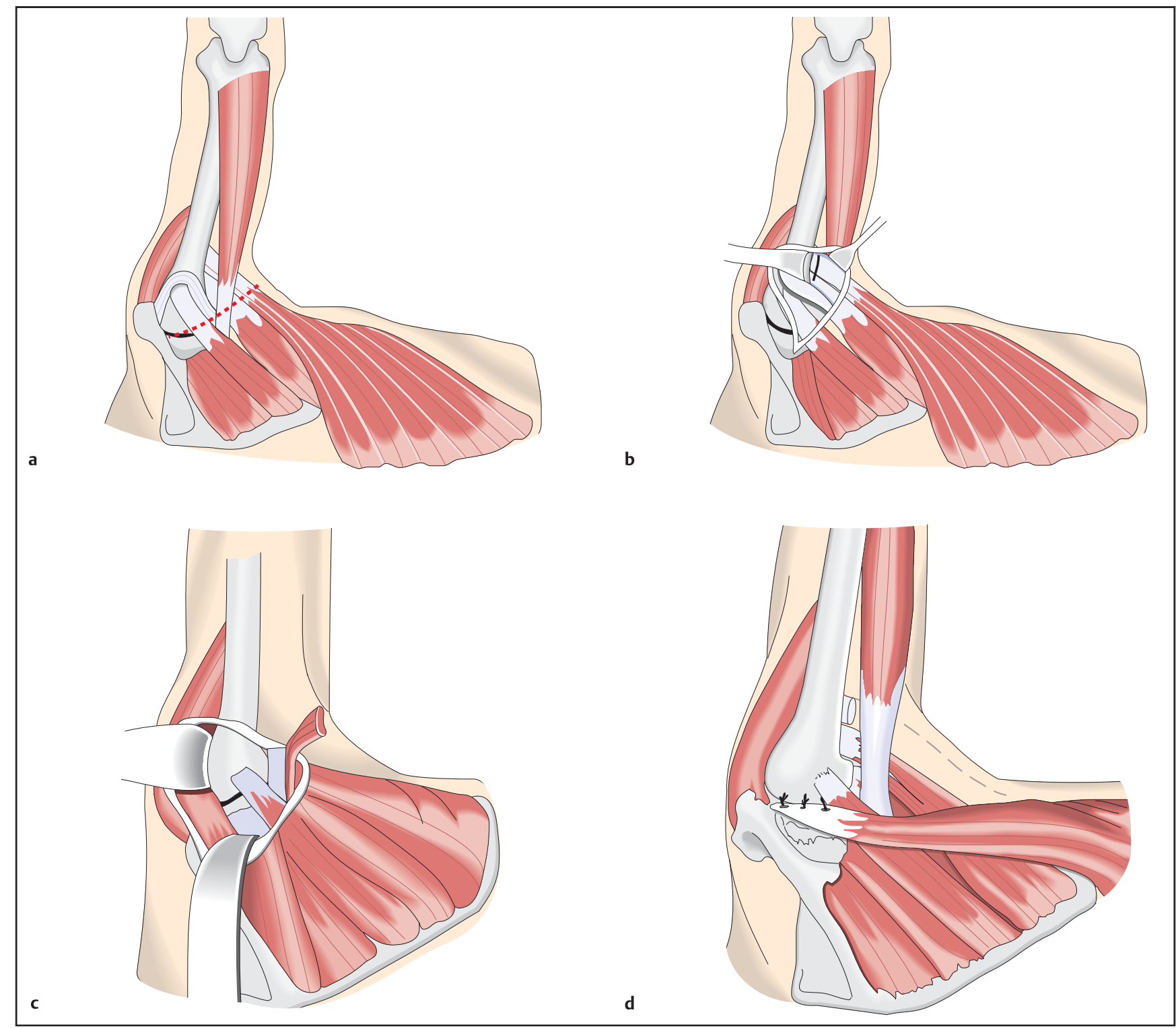

Abb. 8 Latissimus-dorsi-Transfer in Single-Incision-Technik nach Habermeyer/Herzberg. a Hinterer Zugang entlang der Axillarfalte. b Abtrennung der zuvor isolierten Latissimus-dorsi-Sehne am Humerus bei $90^{\circ}$ Abduktion und maximaler Innenrotation des Armes unter Beachtung des Verlaufs des N. radialis. c Darstellung des Rotatorenmanschettendefekts bei außenrotiertem Arm in $90^{\circ}$ Abduktion und abgelöster Latissimus-dorsi-Sehne. Zu beachten ist der N. axillaris, der unmittelbar unterhalb des M. teres minor verläuft. d Mittels dreier Nahtanker im Bereich des Infraspinatussehnenansatzes am Tuberculum majus fixierte transferierte Latissimus-dorsi-Sehne (Mit freundlicher Genehmigung aus: Habermeyer, Lichtenberg, Magosch. Schulterchirurgie. (@) Elsevier GmbH). 


\section{Technik (Abb. 8)}

Bogenförmiger Zugang im Bereich der hinteren Axillarfalte, beginnend am posterioren Arbeitsportal bis in die Axilla reichend auf einer Länge von etwa $12 \mathrm{~cm}$. Bilden eines subkutanen Hautlappens und Abpräparieren von den darunter liegenden Muskelgruppen des M. deltoideus pars spinalis und darunter des M. teres minor, des M. teres major und M. latissimus dorsi sowie des Caput longum des M. trizeps.

Der M. latissimus dorsi wird vom M. teres major durch stumpfes Eingehen zwischen der Sehne des Latissimus dorsi und dem Muskelbauch des Teres major getrennt. Der M. latissimus dorsi wird auf eine Länge von etwa $10 \mathrm{~cm}$ von der seitlichen Thoraxwand abpräpariert. Hierzu Durchführung von tiefen FaszienEntlastungsschnitten. Der proximale Muskelbauch des Latissimus dorsi wird nun zirkulär freipräpariert. Im Gegensatz zur Gerber-Technik ist es nicht notwendig, um einen besseren Schwenkradius für den Muskeltransfer zu erreichen, das Gefäßnervenbündel freizulegen. A. und $V$. thoracodorsalis und $\mathrm{N}$. thoracodorsalis bleiben in situ.

Unter Beachtung der Sicherheitsgrenzen für den $\mathrm{N}$. radialis, der unterhalb der Sehne des M. latissimus dorsal um den Humerusschaft verläuft, wird der Latissimus dorsi bei $90^{\circ}$ Abduktion und bei maximaler Innenrotation am Humerusschaft freipräpariert. Die Sehnenansätze von Teres major und Latissimus dorsi werden am Humerusschaft noch von einander befreit. Dann kann der Latissimus mit seinem sehnigen Ansatz direkt vom Humerusschaft scharf mit dem Skalpell abpräpariert werden.

Im nächsten Schritt erfolgt die Präparation des Sehnenstumpfes des M. latissimus dorsi. Mit einer atraumatischen Naht Stärke $3 \times 0$ wird nun das Sehnenende mit einem Haltefäden eingesäumt, um einen stabilen Sehnennahtrand zu erhalten.

Nun wird unterhalb der Pars spinalis des M. deltoideus und über dem M. teres minor in die Fossa infraspinata eingegangen.

Beachte: Der N. axillaris findet sich unterhalb des $\mathrm{M}$. teres minor und medial des Caput longum M. trizeps. Somit wird der Latissimus dorsi lateral davon in die Fossa infraspinata eingeschwenkt.
Bei maximaler Außenrotation des Armes und bei $90^{\circ}$ Flexion wird nun das Tuberculum innominatum am Humeruskopf dargestellt. Von posterosuperior erreicht man sehr übersichtlich den Humeruskopf und kann den Manschettendefekt bis nach ventral darstellen. Die Bursa subdeltoidea und subacromialis wird exzidiert. Danach wird das Kapsel- und das restliche Sehnengewebe über dem Tuberculum innominatum eröffnet. Unter Rotation Überprüfen der Gelenkflächen. Darstellen der Transitionszone mit der dorsalen und kranialen Facette des Tuberculum majus. Resektion von Restsehnenstumpfgewebe.

Durchführung einer Tuberkuloplastik. Mit dem Luer wird die harte Oberkante des Tuberculum majus abgetragen und abgerundet im Sinne einer umgekehrten Akromioplastik. Auf eine klassische Akromioplastik wird verzichtet, um den Fornix humeri nicht zu schwächen. Anschließend wird eine flache Knochennut im Bereich des hinteren und oberen Tuberculum majus in der Transitionszone auf eine Länge von $3 \mathrm{~cm}$ und eine Breite von $1 \mathrm{~cm}$ geschaffen. Sodann werden 3 Titanankerschrauben in einem Abstand von jeweils $1 \mathrm{~cm}$ zueinander in den $\mathrm{Hu}-$ meruskopf eingedreht und pro Schraube jeweils ein doppeltes Fadenpaar ausgeleitet. Mithilfe der vorgelegten Ankernähte wird nun die Sehne des Latissimus dorsi gegen das Tuberculum innominatum genäht. Hierzu Verwenden einer Mason/Allen-Nahttechnik. Alternativ kann auch eine „double row“-Rekonstruktion durchgeführt werden. Die Haltefäden der Sehne vernäht man zusätzlich.

Insgesamt ist nun die hintere Rotatorenmanschette wieder rekonstruiert und ein Force Couple gegen den ventralen und noch stehenden Subscapularis geschaffen. Dies dient der dynamischen Zentrierung und Kaudalisierung des Humeruskopfes.

Nach Achten auf Bluttrockenheit Einlegen von 2 tiefen Redon-Drainagen, schichtweiser Wundverschluss, Carbostesin-Infiltration eines Lokalanästhetikums subakromial und in die Wundränder, steriler Kompressionsverband. Anlegen eines Orthosoft-Abduktionskissens.

\section{Pectoralis major-Transfer}

Indikation:

- irreparable Subscapularisruptur

- Schmerzen bei anteriorer Elevation
Die Ergebnisse sind am besten bei isolierten Subscapularisläsionen. In Kombination mit einer SSP-Ruptur werden die Ergebnisse deutlich schlechter. Auch zur Verbesserung einer sekundären Subscapularisinsuffizienz nach Schulter-TEP kann der Pectoralis-major-transfer nur einen geringen Beitrag leisten.

Die Eliminierung eines positiven Lift-offoder Belly-Press-Tests lässt sich mit dem Pectoralis-major-Transfer nicht realisieren.

Deltoideopektoraler Zugang beginnend $3 \mathrm{~cm}$ oberhalb des mittleren Drittels der Clavicula bis zum Ansatz des Deltamuskels am Humerus. Subkutanes Freilegen der anterioren Portion des M. deltoideus und der klavikulären sowie der oberen sternalen Portion des M. pectoralis major.

In der Technik nach Rockwood und Wirth werden beide - sternale und klavikuläre - Anteile des Pectoralis an der Crista humeri abgelöst und über die Conjoint Tendons am Processus coracoideus vorbei geleitet. Die Re-Insertion erfolgt entweder transossär oder mittels Nahtanker lateral des Sulcus bicipitalis.

In der Technik nach Resch nimmt man den sternalen Anteil zum Verschwenken. Die Trennung der beiden Anteile erfolgt ansatznah an der Crista humeri. Man kann die Sehne entweder direkt am Knochen oder mit einer Knochenschuppe ablösen. Die beiden Anteile sind dann stumpf voneinander $\mathrm{zu}$ trennen, auch um das Gefäß-Nerven-Bündel zu schonen. Unterhalb der Coracoids wird dann eine Passage präpariert, wobei auf den Verlauf des N. musculocutaneus geachtet werden muss. Ferner muss die Passage breit genug für den zu transferierenden Muskel sein, damit Stauungen und Kompressionen vermieden werden können. Die Re-Insertion erfolgt am Tuberculum minus mittels Nahtankern oder transossär.

Postoperativ folgt eine Ruhigstellung in der Armschlinge für 4 Wochen mit rein passiver Beübung der Abduktion, Flexion und Innenrotation.

\section{Zusammenfassung}

Die Behandlung der Rotatorenmanschettenläsionen nimmt heute eine wichtige sozioökonomische Rolle ein. Aus diesem Grund kann und muss die Diagnostik und Therapie dieser Läsionen sehr diffe- 
renziert und individualisiert erfolgen, was einer Vielzahl diagnostischer und therapeutisch operativer Optionen den Weg bereitet. Aus diesem Konzert der Möglichkeiten das optimale Konzept für den Patienten mit seinen individualisierten Ansprüchen und Nöten herauszufiltern, ist die Aufgabe des modernen, auf Schulterprobleme spezialisierten Orthopäden.

\section{Literatur}

${ }^{1}$ Deutsche Vereinigung für Schulter- und Ellenbogenchirurgie (DVSE) e.V. Untersuchungstechniken des Schultergelenkes. Obere Extremität 2012; 7 (Suppl. 1) : 1-67

${ }^{2}$ Habermeyer P, Krieter C, Tang KL et al. A new arthroscopic classification of articular-sided supraspinatus footprint lesions: a prospective comparison with Snyder's and Ellman's classification. J Shoulder Elbow Surg 2008; 17: 909-913

${ }^{3}$ Cofield RH, Parvizi J, Hoffmeyer PJ et al. Surgical repair of chronic rotator cuff tears. A prospective long-term study. J Bone Joint Surg [Am] 2001; 83: 71-77

4 Gladstone JN, Bishop JY, Lo IK et al. Fatty infiltration and atrophy of the rotator cuff do no improve after rotator cuff repair and correlate with poor functional outcome. Am J Sports Med 2007; 35: 719-728

${ }^{5}$ Matthews TJ, Hand GC, Rees JL et al. Pathology of the torn rotator cuff tendon. Reduction in potential for repair as tear size increases. J Bone Joint Surg [Br] 2006; 88: 489-495

${ }^{6}$ Mormino MA, Gross RM, McCarthy JA. Captured shoulder: a complication of rotator cuff surgery. Arthroscopy 1996; 12: 457-461

${ }^{7}$ Berg EE, Ciullo JV. Heterotopic ossification after acromioplasty and distal clavicle resection. J Shoulder Elbow Surg 1995; 4: 188-193
8 Lichtenberg S, Liem D, Magosch P et al. Influence of tendon healing after arthroscopic rotator cuff repair on clinical outcome using single-row Mason-Allen suture technique: a prospective, MRI controlled study. Knee Surg Sports Traumatol Arthrosc 2006; 14: 12001206

9 Castagna A, Delle Rose G, Conti M et al. Predictive factors of subtle residual shoulder symptoms after transtendinous arthroscopic cuff repair: a clinical study. Am J Sports Med 2009; 37: 103-108

10 Yamaguchi K, Tetro AM, Blam $O$ et al. Natural history of asymptomatic rotator cuff tears: a longitudinal analysis of asymptomatic tears detected sonographically. J Shoulder Elbow Surg 2001; 10: 199-203

11 Lo IK, Burkhart SS. The comma sign: an arthroscopic guide to the torn subscapularis tendon. Arthroscopy 2003; 19: 334-337

12 Boileau P, Brassart N, Watkinson DJ et al. Arthroscopic repair of full-thickness tears of the supraspinatus: does the tendon really heal? J Bone Joint Surg [Am] 2005; 87: 1229-1240

13 Cole BJ, McCarty 3rd LP, Kang RW et al. Arthroscopic rotator cuff repair: prospective functional outcome and repair integrity at minimum 2-year follow-up. J Shoulder Elbow Surg 2007; 16: 579-585

${ }^{14}$ Liem D, Bartl C, Lichtenberg S et al. Clinical outcome and tendon integrity of arthroscopic versus mini-open supraspinatus tendon repair: a magnetic resonance imaging-controlled matched-pair analysis. Arthroscopy 2007: 23: 514-521

15 Wilson F, Hinov V, Adams G. Arthroscopic repair of full-thickness tears of the rotator cuff: 2 - to 14 -year follow-up. Arthroscopy 2002; 18: $136-144$

${ }^{16}$ Lee E, Bishop JY, Braman JP et al. Outcomes after arthroscopic rotator cuff repairs. J Shoulder Elbow Surg 2007; 16: 1-5

17 Burkhart SS, Danaceau SM, Pearce Jr CE. Arthroscopic rotator cuff repair: Analysis of results by tear size and by repair technique- margin convergence versus direct tendonto-bone repair. Arthroscopy 2001; 17: $905-$ 912

18 Galatz LM, Ball CM, Teefey SA et al. The outcome and repair integrity of completely arthroscopically repaired large and massive rotator cuff tears. J Bone Joint Surg [Am] 2004; 86: 219-224

${ }^{19}$ Milgrom C, Schaffler M, Gilbert S et al. Rotatorcuff changes in asymptomatic adults. The effect of age, hand dominance and gender. J Bone Joint Surg [Br] 1995; 77: 296-298

${ }^{20}$ Thomazeau H, Lucs C. Comment in: Gazielly DF, Gleyze, P,Thomas T, eds. The Cuff. Amsterdam, Oxford, Paris, New York, Tokyo: Elsevier; 1997: 92-94

${ }^{21}$ Goutallier D, Postel JM, Lavau L et al. [Impact of fatty degeneration of the suparspinatus and infraspinatus msucles on the prognosis of surgical repair of the rotator cuff]. Rev Chir Orthop Reparatrice Appar Mot 1999; 85: 668-676

\section{Dr. med. Sven Lichtenberg}

Orthopädie und Unfallchirurgie Sportmedizin

Schulter- und Ellenbogenchirurgie

Dr. med. Petra Magosch

Orthopädie und Unfallchirurgie

Mediz. Informatik

Prof. Dr. med. Peter Habermeyer

Chirurg, Unfallchirurg

Orthopädie und Unfallchirurgie

Schulter- und Ellenbogenchirurgie

ATOS-Klinik Heidelberg

Bismarckstraße 9-15

69115 Heidelberg

lichtenberg@atos.de 